\title{
Spectral properties of X-ray bright variable sources in the Taurus molecular cloud ${ }^{\star}$
}

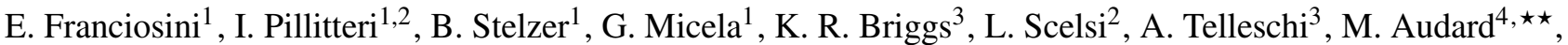 \\ F. Palla ${ }^{5}$, and M. Güdel ${ }^{3}$ \\ 1 INAF - Osservatorio Astronomico di Palermo, Piazza del Parlamento 1, 90134 Palermo, Italy \\ e-mail: francio@astropa.unipa.it \\ 2 Dipartimento di Scienze Fisiche ed Astronomiche, Università di Palermo, Piazza del Parlamento 1, 90134 Palermo, Italy \\ 3 Paul Scherrer Institute, Würenlingen and Villigen, 5232 Villigen PSI, Switzerland \\ 4 Columbia Astrophysics Laboratory, Mail Code 5247, 550 West 120th Street, New York, NY 10027, USA \\ 5 INAF - Osservatorio Astrofisico di Arcetri, Largo E. Fermi 5, 50125 Firenze, Italy
}

Received 10 October 2006 / Accepted 12 November 2006

\section{ABSTRACT}

\begin{abstract}
Aims. We analyze 19 bright variable X-ray sources detected in the XMM-Newton Extended Survey of the Taurus Molecular Cloud (XEST), to characterize the variations with time of their coronal properties and to derive information on the X-ray emitting structures. Methods. We performed time-resolved spectroscopy of the EPIC PN and MOS spectra of the XEST sources, using a model with one or two thermal components, and we used the time evolution of the temperatures and emission measures during the decay phase of flares to derive the size of the flaring loops.

Results. The light curves of the selected sources show different types of variability: flares, long-lasting decay or rise through the whole observation, and slow modulation or complex flare-like variability. Spectral analysis shows typical quiescent plasma temperatures of 5-10 MK and 15-35 MK; the cool component generally remains constant, while the observed flux changes are due to variations of the hot component. During flares the plasma reaches temperatures up to $100 \mathrm{MK}$ and luminosities up to $\sim 10^{31} \mathrm{erg} \mathrm{s}^{-1}$. Loop sizes inferred from flare analysis are generally smaller than or comparable to the stellar radius.
\end{abstract}

Key words. stars: coronae - stars: late-type - stars: pre-main sequence - X-rays: stars

\section{Introduction}

Young pre-main sequence (PMS) stars are known to exhibit strong X-ray emission at levels orders of magnitude higher than generally observed in older active stars (Feigelson \& Montmerle 1999; Favata \& Micela 2003; Güdel 2004). Furthermore, X-ray time variability is almost always detected in PMS stars, very often in the form of flares, with a shape similar to solar flares, or of slow modulation due, e.g., to rotation (Feigelson \& Montmerle 1999; Wolk et al. 2005; Flaccomio et al. 2005). On the Sun, the X-ray time variability is due to flare activity on timescales of a few hours, to the appeareance of new active regions combined with the surface rotation (timescales of a few weeks), and to the 11-yr solar activity cycle. The flare activity observed in PMS stars suggests that a scaled version of the solar corona should be present in these objects. The plasma magnetically confined in loop-like structures would be heated by mechanisms similar to those present in the solar corona, and would release the energy in the form of flares. However, observations

* Table 2 is only available in electronic form at http: //www . aanda. org

$\star \star$ Present address: Integral Science Data Centre, Ch. d'Ecogia 16, 1290 Versoix, Switzerland \& Geneva Observatory, Ch. des Maillettes 51, 1290 Sauverny, Switzerland. show that Classical T Tauri stars (CTTS), characterized by accretion from the circumstellar disk, are statistically X-ray underluminous with respect to weak-lined T Tauri stars (WTTS), where disks are absent or weak (Stelzer \& Neuhäuser 2001; Flaccomio et al. 2003; Preibisch et al. 2005; Franciosini et al. 2006; Telleschi et al. 2007b). Moreover, high-resolution spectroscopy of the CTTS TW Hya (Kastner et al. 2002; Stelzer \& Schmitt 2004) and BP Tau (Schmitt et al. 2005) indicate the presence of very high densities $\left(n_{\mathrm{e}} \gtrsim 10^{12} \mathrm{~cm}^{-3}\right)$ and a dominant cool plasma component at $3 \mathrm{MK}$ in TW Hya that have been attributed to emission from plasma heated by an accretion shock. Enhanced X-ray emission and/or significant spectral variations have been observed during accretion outbursts in V1647 Ori and V1118 Ori (Kastner et al. 2004, 2006; Grosso et al. 2004; Audard et al. 2005). These results suggest that accretion may play a role in the X-ray emission process, either by influencing the magnetic structure, or by providing an alternative $\mathrm{X}$-ray production mechanism.

The study of the time variability of the X-ray emission from PMS stars allows us to gain insights into the structure and the heating mechanisms of stellar coronae. In particular, the analysis of flares constitutes a diagnostic tool to infer the size of the X-ray emitting structures. Recently, Favata et al. (2005) studied a sample of intense flares observed in the Orion Nebula Cluster 
as part of the Chandra Orion Ultradeep Project (COUP), finding that flares on PMS stars occur both in small loops, of size less than a stellar radius, similar to older active stars, and in large loops, up to $10-20 R_{\star}$, likely connecting the stellar surface and the circumstellar disk.

In this paper we study the X-ray properties of a sample of PMS stars of the Taurus molecular cloud (TMC) showing significant time variability. The TMC is one of the nearest regions of star formation ( $d=140 \mathrm{pc}$ ), containing $\sim 340$ known members in an area $\sim 100$ square degrees large, and is characterized by a low stellar density $\left(1-10 \mathrm{pc}^{-3}\right)$, by the lack of massive stars, and by a significant fraction of binary or multiple systems. Star formation appears to have occurred in several epochs during the last 10 Myr. Our work complements the study by Stelzer et al. (2007), who analyze the X-ray time variability of TMC sources from a statistical point of view, and derive the frequency and energy distribution of flaring events. In our paper we concentrate on the time-dependent spectral analysis of the brightest sources, showing both flares and other kinds of variability, to investigate the changes of the plasma parameters and to derive information on the X-ray emitting structures.

The paper is organized as follows. In Sect. 2 we describe the target selection and the data analysis. In Sect. 3 we present the results obtained for TMC members, whereas in Sect. 4 we present the variability of three sources not related to the TMC. Discussion and conclusions are given in Sect. 5.

\section{Source selection and analysis}

\subsection{Observations}

Our study is based on the data obtained from the XMM-Newton Extended Survey of the Taurus Molecular Cloud (XEST), a wide $\mathrm{X}$-ray survey performed with the XMM-Newton satellite aimed at studying the properties of young PMS stars in the TMC (Güdel et al. 2007a). The survey consists of 19 fields of $\sim 33 \mathrm{ks}$ exposure each, covering the densest regions of the cloud, plus 9 archival exposures lasting up to $\sim 130 \mathrm{ks}$; the total area covered by the survey is about 5 square degrees. A detailed description of the survey, including details on the primary reduction of the raw EPIC MOS and PN datasets, on the source detection procedure, and on the definition of the extraction regions for the source and background light curves and spectra is reported in Güdel et al. (2007a).

The work presented here uses data from all XEST fields except for XEST-01 and XEST-25 that are dedicated to separate projects (Güdel et al. 2007b; Grosso et al., in preparation). We also excluded the classical $\mathrm{T}$ Tauri star SU Aur (source XEST-26-067) that is discussed in detail in a separate paper (Franciosini et al. 2007). We note that two of the fields, namely XEST-23 and XEST-24, correspond to two consecutive observations with the same pointing position, with a duration of $\sim 70$ and $40 \mathrm{ks}$, respectively, separated by $\sim 5 \mathrm{ks}$. Sources detected in both exposures have been treated as a single source in the following analysis, with the two exposures added together.

\subsection{Analysis of light curves and sample selection}

To select the sample of sources for this study, we first extracted, for all detected X-ray sources, the PN and MOS source and background photons in the energy band $0.3-7.3 \mathrm{keV}$, using the extraction regions defined by Güdel et al. (2007a). To have a continuous time coverage for each observation, without gaps in the light curves, we chose not to filter out the periods of high background count rate due to proton flares. We then limited our analysis to sources with at least 1500 net counts in the PN exposures (or in the MOS exposures in the cases where the PN was not available), to have sufficiently high count statistics for good time-resolved spectral analysis. This selection necessarily biases our study towards the X-ray brightest and likely most active sources.

For these sources, we extracted the PN (or MOS) light curves, and applied the Maximum Likelihood Blocks (MLB) method described by Stelzer et al. (2007) to them, to identify variable sources and to define the intervals to be used for timedependent spectroscopy. The MLB algorithm divides the full exposure into time intervals (blocks) where the source is assumed to be constant; the boundaries of the time blocks are chosen such that between two consecutive blocks the mean count rate changes by more than a given significance threshold. The significance threshold for the change points, at the $99 \%$ level, was determined through simulations of constant rate light curves (Flaccomio et al., in preparation). We set a minimum number of 750 net source photons for each block, to have enough counts in each spectrum to perform a reliable spectral analysis. We note that this choice does not allow us to examine rapid variations, small-amplitude flaring events, and low-level variability.

The variability of the background in many XEST fields was significant and was taken into account using the following procedure: the MLB algorithm was used to split the background light curve into blocks of constant count rate level; for each of these blocks, the background level was scaled to the source extraction area to yield the number of expected background photons in the source area for the given time interval. The resulting number of photons is then removed from the source event file uniformly across each background block. The result is a "sourceonly" event file, i.e., a background-subtracted photon time series for the source.

Using the MLB results, we restricted our sample by considering only those sources whose light curves showed significant variability, i.e., those that have been divided by the algorithm in at least two blocks with mean count rates differing by more than $3 \sigma$. This selection process led to a sample of 16 variable X-ray sources associated with known PMS members of the TMC, plus additional three sources not related to the region or that are not confirmed members of the TMC. The weakest source in the final sample is JH 108, with 2500 net PN counts. The main properties of the selected sources are given in Table 1 . The three non-members will be discussed separately in Sect. 4. The 16 TMC members are mostly late-K and early-M stars; five of them are classified as CTTS, ten as WTTS, and one is a Herbig Ae star. Six of these sources (including the Herbig Ae star) are binary or multiple systems unresolved in $\mathrm{X}$-rays with XMM-Newton.

\subsection{Time-resolved spectroscopy}

We have performed a time-dependent spectral analysis of the selected sources, to study the changes with time of the plasma characteristics, i.e., temperature and emission measure (EM), that give us information on the origin of the emission, and to derive, in the case of flares, information on the size of the flaring region from flare modeling.

PN and MOS spectra for each source and each time block have been extracted from the source and background event files. For the response matrices, we used the appropriate canned response matrix files for PN and MOS, and the ancillary response files produced for each individual source (see Güdel et al. 2007a, for details). The PN and MOS spectra have been rebinned in 
Table 1. Properties of the sample of XEST variable sources analyzed in this paper. Stellar parameters are taken from Güdel et al. (2007a). In the last column we give a classification of the observed variability.

\begin{tabular}{llllll}
\hline \hline XEST ID & Optical ID & Sp. T. & TTS class. & $R_{\star} / R_{\odot}$ & Variab. type \\
\hline $04-016$ & V830 Tau & K7 & WTTS & 1.79 & slow decay \\
$09-026$ & HQ Tau AB & $\ldots$ & WTTS & $\ldots$ & slow decay \\
$11-057$ & FS Tau AC & M0+M3.5 & CTTS+CTTS & 0.93 & slow decay \\
$12-040$ & DN Tau & M0 & CTTS & 2.25 & slow modulation \\
$15-040$ & DH Tau AB & M1 & CTTS & 1.82 & slow decay \\
$17-066$ & JH 108 & M1 & WTTS & 1.32 & atypical flare \\
$21-039$ & HD 283572 & G5 & WTTS & 2.56 & slow modulation \\
$22-047$ & XZ Tau AB & M2+M3.5 & CTTS+CTTS & 1.18 & slow rise \\
$22-089$ & L1551 51 & K7 & WTTS & 1.39 & slow decay \\
$23-032 / 24-028$ & V410 Tau ABC & K4 & WTTS & 2.31 & atypical flare \\
$23-047 / 24-040$ & V892 Tau & B9 & Herbig Ae & 2.66 & atypical flare \\
$23-050 / 24-042$ & V410 X7 & M0.75 & WTTS & 1.69 & flares \\
$23-056 / 24-047$ & Hubble 4 & K7 & WTTS & 3.33 & complex variab. \\
$23-074 / 24-061$ & V819 Tau AB & K7 & WTTS & 1.93 & flare \\
$26-072$ & HBC 427 & K7 & WTTS & 1.85 & flare \\
$28-100$ & BP Tau & K7 & CTTS & 1.97 & flare \\
\hline $05-031$ & HD 283810 & K5V & & & flare \\
$16-031$ & 2M J04195676+2714488 & $\ldots$ & & & flares \\
$22-024$ & HD 285845 & G6 & & & complex variab. \\
\hline
\end{tabular}

order to have at least 15 counts per bin, and have been jointly fitted in XSPEC v.11.3.2, using a thermal VAPEC model with one or two temperature components, plus a common photoelectric absorption component. The hydrogen column density $N_{\mathrm{H}}$ was left as a free parameter, except in a few cases where it was not constrained by the fit, and it was therefore fixed to a value derived for other blocks of the same source. Abundances were kept fixed, with their values following a pattern derived from estimates of coronal abundances of X-ray active young stars ${ }^{1}$ (Scelsi et al. 2005; Telleschi et al. 2005; Argiroffi et al. 2004). The best-fit results are reported in Table 2; errors are $90 \%$ confidence intervals for one interesting parameter.

Figures 1-9 show the light curves and spectral parameters derived for TMC sources; the results for TMC non-members are shown in Figs. 10-11. For each source, we plot in the top panel the PN (or MOS) background-subtracted light curve and the background light curve, while in the other panels we show the evolution of the best-fit temperatures and EMs and of the hydrogen column density. In Figs. 2, 3, 5, and 11 we also show, in the right panels, the evolution of the plasma temperature and emission measure used for flare analysis.

\subsection{Flare modeling}

Flare light curves contain information on heating and cooling parameters and indirectly on the density and geometry of the flaring sources. As stellar flare observations are not spatially resolved, various techniques have been developed to use the light curves and measurable quantities such as the emission measure or the electron temperature to deduce flare source parameters (see review by Güdel 2004). Each of these methods introduces simplifications and makes various assumptions on the heating and cooling processes, often adopted by analogy with solar flares. A particularly straightforward physical model has been developed by Reale et al. (1997) that derives the heating fraction and the magnetic loop length from hydrodynamic

1 The abundances used, relative to the solar abundances by Anders \& Grevesse (1989), are: $\mathrm{C}=0.45, \mathrm{~N}=0.788, \mathrm{O}=0.426, \mathrm{Ne}=0.832$, $\mathrm{Mg}=0.263, \mathrm{Al}=0.5, \mathrm{Si}=0.309, \mathrm{~S}=0.417, \mathrm{Ar}=0.55, \mathrm{Ca}=0.195$, $\mathrm{Fe}=0.195$, and $\mathrm{Ni}=0.195$. considerations, using only the run of flare temperature and emission measure. Its advantage is its simple application to stellar observations; it has also been tested on moderate single-loop solar flares. It is, on the other hand, not applicable to flares occurring in magnetic arcades as in gradual solar flares, where a large number of magnetic structures are ignited and cool in sequence. Then, both the rise and the decay times are essentially determined by the history of the heating energy release rather than by cooling physics (e.g., Pneuman 1982; Kopp \& Poletto 1984). Comparative studies have been presented in Güdel et al. (2004) and Reale et al. (2004) for an exceptionally strong flare on the nearby active star Proxima Centauri. We are not in a position to characterize flares on T Tau stars here. We henceforth will adopt the model of Reale et al. (1997) to discuss possible systematics in our results.

In the case of a single flaring loop decaying freely after an initial impulsive heating, Serio et al. (1991) showed that the loop semi-length $L$ is related to the thermodynamic decay time $\tau_{\text {th }}$ by the relation:

$L=\frac{\tau_{\text {th }} \sqrt{T_{\max }}}{3.7 \times 10^{-4}}$

where $T_{\max }$ is the maximum temperature of the flaring plasma. However, if significant heating is present also during the decay phase, the flare decay time will be longer than $\tau_{\text {th }}$, and the above equation would lead to an overestimate of the loop length. Using hydrodynamical simulations of flaring loops taking into account the presence of prolonged heating, Reale et al. (1997) showed that the slope $\zeta$ of the flare decay path in a diagram of $\log T$ vs. $\log n_{\mathrm{e}}$ (or equivalently $\log \sqrt{\mathrm{EM}}$ ) is a diagnostic of the presence of residual heating during the flare decay. The loop semilength $L$ can then be derived from $\zeta$ and the observed flare decay timescale $\tau_{\mathrm{lc}}$ using the following relation:

$L=\frac{\tau_{\mathrm{lc}} \sqrt{T_{\max }}}{3.7 \times 10^{-4} F(\zeta)}$,

where $F(\zeta)$ is the ratio between the observed and thermodynamic decay times, which depends on the amount of residual heating. $T_{\max }$, as in Eq. (1), is the maximum temperature in the 

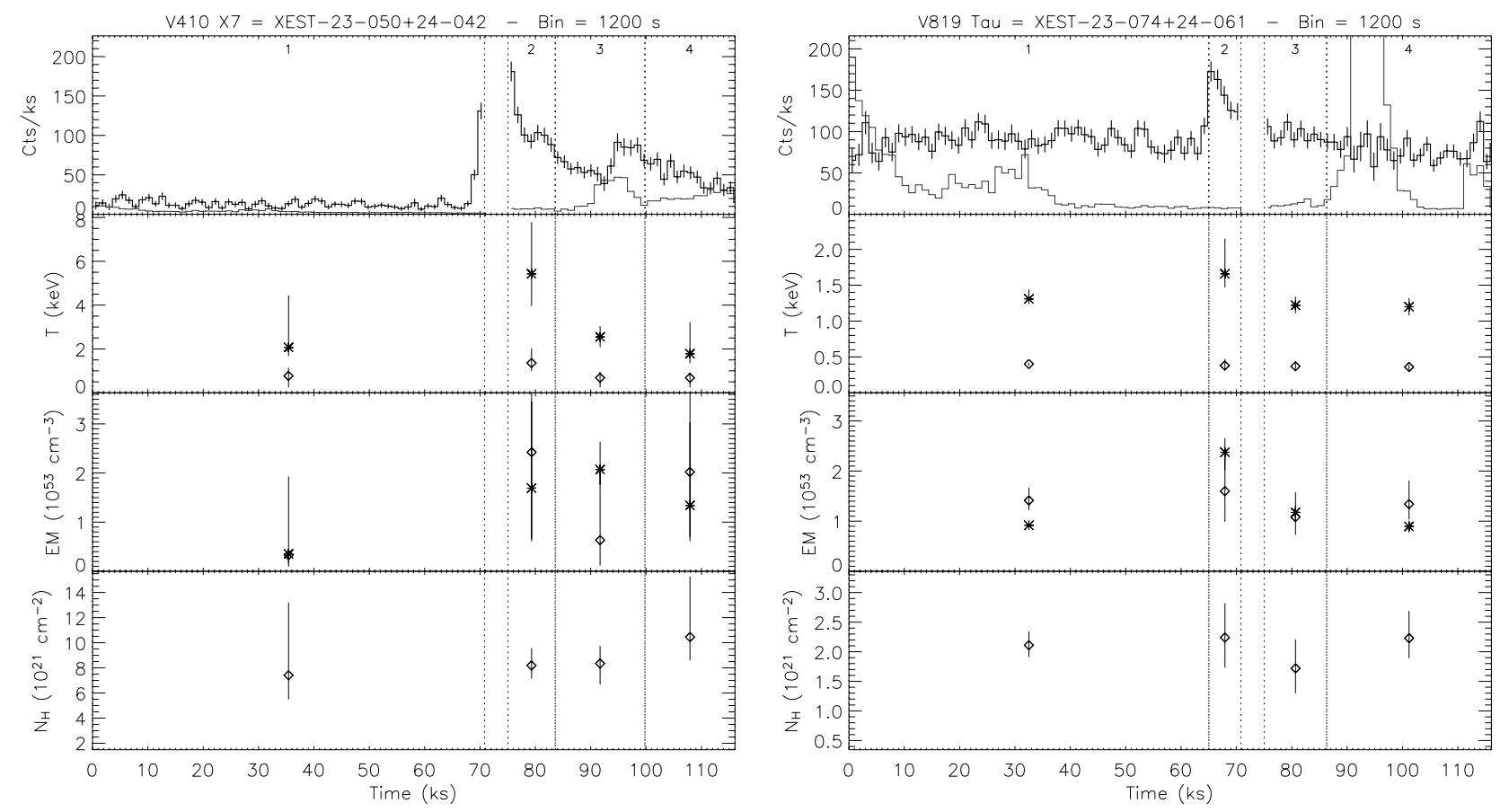

Fig. 1. Light curves and spectral fitting results for the flaring TMC sources V410 X7 (XEST-23-050/24-042) and V819 Tau (XEST-23-074/24-061). In the top panels, the background-subtracted PN light curve of each source is plotted with a thick solid line, while the thin solid line shows the background light curve, scaled to the source area. The vertical dotted lines mark the time intervals, derived from the MLB analysis, used for the time-resolved spectroscopy. In the other panels we show, from top to bottom, the time evolution of the best-fit temperatures, emission measures, and hydrogen column density. The temperatures and EMs of the cool and hot components are indicated by diamonds and asterisks, respectively.

flaring loop, that can be derived from the flare peak temperature $T_{\text {obs }}$ obtained from the spectral fits. The expression of $F(\zeta)$ and the relationship between $T_{\max }$ and $T_{\text {obs }}$ depend on the instrumental response. In the case of EPIC PN we have (Giardino et al. 2006):

$T_{\max }=0.13 T_{\mathrm{obs}}^{1.16}$

and

$F(\zeta)=1.36+\frac{0.51}{\zeta-0.35}$

The expression of $F(\zeta)$ is valid only for $0.35<\zeta \leq 1.6$, where the lower limit corresponds to the case where the heating timescale is comparable to the flare decay timescale, while the upper limit corresponds to a freely-decaying loop with no residual heating after the impulsive energy release. The above formulae are calibrated on the EPIC PN response, however, they give a correct order of magnitude estimate of the loop length also in the case of MOS, given the similarity of the instrumental responses and the wide spectral band used. We have therefore also applied them to the analysis of the flare of HBC 427, for which PN is not available.

\section{Sources associated with TMC members}

We have divided the sources associated with TMC members into three classes according to the type of variability observed in their light curves: (i) sources showing evident flares, either with a "classical" shape, with fast rise and slower decay, or with a gradual rise and/or a symmetrical shape; (ii) sources with slow prolonged decay or rise of the count rate; and (iii) sources with slow modulation or complex variability that could be due to superimposed flaring events.

\subsection{Flares}

Seven of the TMC sources show evident flares in their light curve. Four of them (V410 X7, V819 Tau, HBC 427, and BP Tau) show the typical flare behavior, characterized by a rapid increase of the count rate and of the plasma temperature, followed by a slower decay. Their light curves and the time evolution of the plasma parameters are shown in Figs. 1-3. The other three sources (JH 108, V892 Tau, and V410 Tau) show peculiar flaring variability, i.e., flares that are characterized by a gradual rise phase with a flat top and/or a nearly symmetrical shape. Their light curves and evolution of the plasma parameters are shown in Figs. 4 and 5.

\subsubsection{V410 X7 (XEST-23-050/24-042)}

The light curve of V410 X7 (XEST-23-050/24-042, M0.75, WTTS) shows two flares that occurred after an initial quiescent period lasting for $\sim 65 \mathrm{ks}$. Unfortunately, the XEST-23 exposure stopped just after the start of the rise phase of the first flare, while at the beginning of the XEST-24 exposure the flare was already decaying; therefore the flare peak was not observed. The flare is very strong, with an increase of the count rate by more than an order of magnitude. The decay phase is initially rapid, with an e-folding timescale of $\sim 2.1 \mathrm{ks}$; then a slower decay is observed, after a small bump occurring about $5 \mathrm{ks}$ after the start of the XEST-24 exposure. A second smaller flare, with a decay efolding time of $\sim 20 \mathrm{ks}$, is superimposed on the late decay phase. As mentioned in Sect. 2.2, our choice of the block size does not allow us to derive the plasma characteristics of this second flare separately from the decay of the first one.

The quiescent emission has temperatures of $\sim 9$ and $24 \mathrm{MK}$ with similar EMs. The first flare reached a temperature $>60 \mathrm{MK}$ at the peak, as estimated from the best-fit temperature of 

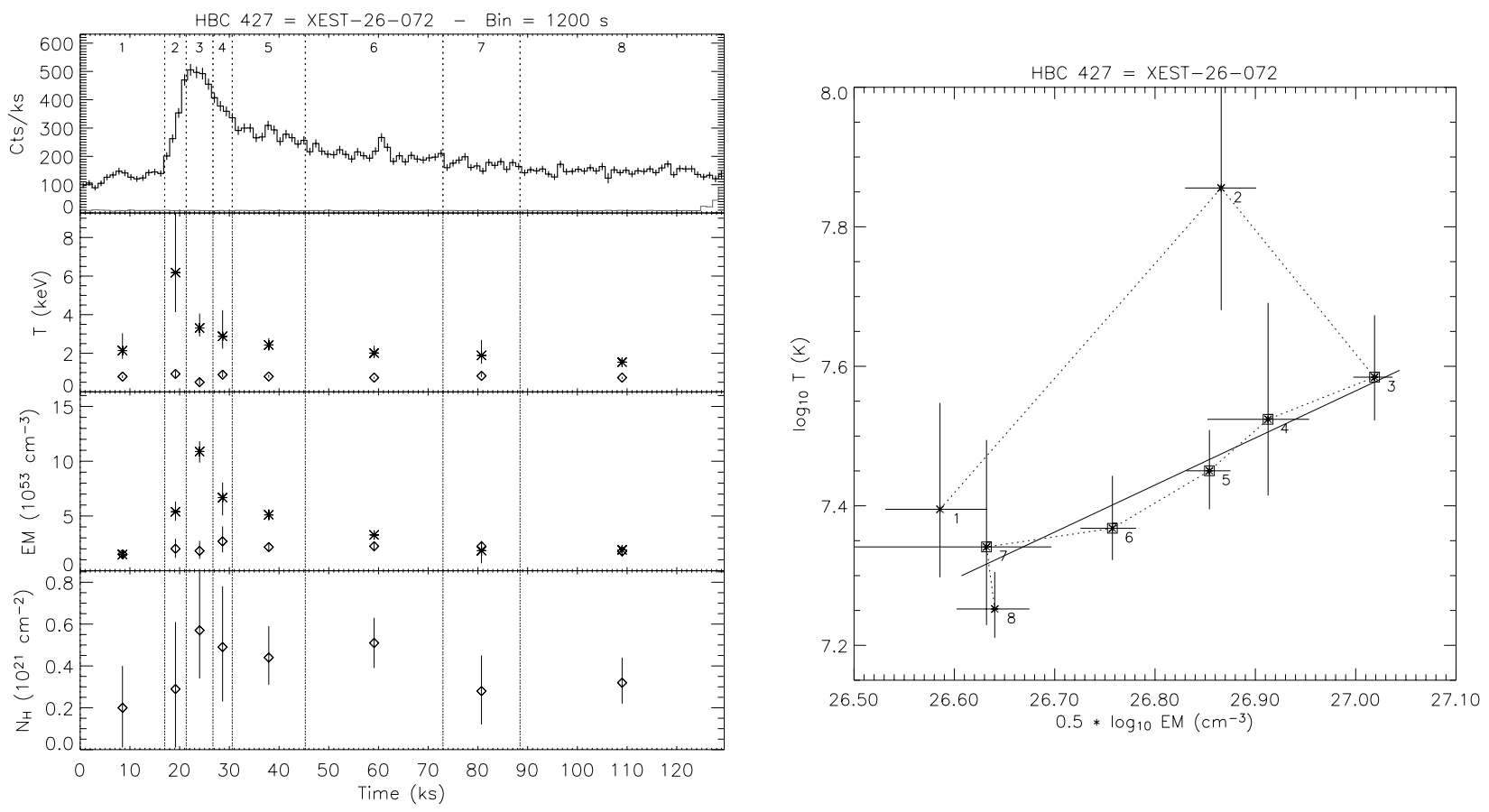

Fig. 2. Left: light curve and spectral fitting results for HBC 427 (XEST-26-072). Panels are the same as in Fig. 1. Note that in this case we show the combined MOS1+MOS2 light curve, the PN being not available. Right: evolution of the best-fit temperature and EM of the hot component for HBC 427. To better clarify the trend with time of the parameters, the points are connected by dotted lines. The thick solid line shows the linear fitting to points \#3 to \#7, corresponding to the flare decay; for clarity they are marked with open squares.

block \#2 where the flare is already decaying. Taking this value as peak temperature and the decay time of $2.1 \mathrm{ks}$, and applying to this flare the formula for a freely-decaying loop (Eq. (1)), we can put a lower limit on the loop semi-length of $L \geq 5 \times 10^{10} \mathrm{~cm}$, corresponding to $\sim 0.4 R_{\star}$.

\subsubsection{V819 Tau (XEST-23-074/24-061)}

V819 Tau (XEST-23-074/24-061) is a binary WTTS with spectral type K7. It showed a small impulsive flare at the end of the XEST-23 exposure, with a factor of 2 increase in the count rate, whose decay continues in the XEST-24 observation. The decay phase has an e-folding time of $\sim 10 \mathrm{ks}$. The flare is not very hot, with $T_{2}=20 \mathrm{MK}$ in block \#2, not much higher than the quiescent value of $\sim 15 \mathrm{MK}$, although the true peak temperature is likely hotter since this time interval also includes a significant part of the decay phase. We also note that the cool component remained constant, within the errors, for the whole observation. We can obtain a rough estimate of the loop semi-length using the temperatures and EMs of the hot component in blocks \#2 and \#3, from which we derive $\zeta \sim 0.9$ and $L \sim 7 \times 10^{10} \mathrm{~cm}$, corresponding to $0.5 R_{\star}$.

\subsubsection{HBC 427 (XEST-26-072)}

During the XMM-Newton observation, the K7 WTTS HBC 427 (XEST-26-072) underwent a strong long-duration flare that covered nearly the entire exposure time of $\sim 130 \mathrm{ks}$. After a short initial quiescent phase, the count rate increased by a factor of 5 in $\sim 6 \mathrm{ks}$, then it decreased slowly, returning to a level similar to the preflare one after $\sim 70 \mathrm{ks}$. The decay e-folding time is $20.2 \pm$ $1.0 \mathrm{ks}$. The time evolution of the plasma parameters follows the typical behavior observed in flares, with the temperature peaking during the rise phase and the emission measure peaking at the flare peak. The quiescent emission has temperatures of $\sim 8$ and $25 \mathrm{MK}$ with equal EMs. While the temperature and EM of the coolest component does not change significantly during the flare, the hot component reaches a temperature as high as $\sim 72 \mathrm{MK}$; therefore, for the flare analysis we will assume that the flaring plasma is described by the hot component.

In the right panel of Fig. 2 we show the evolution of the flare in the $T$ vs. $\sqrt{\mathrm{EM}}$ plane. The maximum loop temperature, derived from Eq. (3), is $\sim 168 \mathrm{MK}$. From the linear fitting of the points from block \#3 to block \#7 we find a slope $\zeta=0.67 \pm$ 0.09 . This value indicates the presence of significant residual heating after the initial ignition. Combining the observed decay time with the above value of $\zeta$, from Eq. (2) we derive $L=2.4 \pm$ $0.4 \times 10^{11} \mathrm{~cm}$, i.e., $\sim 2$ stellar radii. From the EM of the hot component in block \#2 we can estimate the mean electron density in the loop at the peak of the flare, assuming a loop with typical aspect ratio $R_{\text {loop }} / L=0.1$, as commonly observed in solar flares: we find $n_{\mathrm{e}} \sim 2 \times 10^{10} \mathrm{~cm}^{-3}$, consistent with the values found for the COUP flares with similar loop size (Favata et al. 2005).

\subsubsection{BP Tau (XEST-28-100)}

BP Tau (XEST-28-100) is a K7 CTTS. Its light curve shows a flare beginning $\sim 15 \mathrm{ks}$ after the start of the observation, with an increase of the count rate by a factor of $\sim 2.5$ in $\sim 5 \mathrm{ks}$ and a total duration of $\sim 35 \mathrm{ks}$. The e-folding decay time is $10.4 \pm$ $0.7 \mathrm{ks}$. After the flare the X-ray emission shows low-level variability until the end of the observation. As for HBC 427, BP Tau shows the typical flare behavior of the plasma parameters, with the temperature peaking in the rise phase before the EM. The quiescent emission before the flare has temperatures of $\sim 4$ and $18 \mathrm{MK}$ with $\mathrm{EM}_{2} / \mathrm{EM}_{1}=1.5$; the temperature of the cooler component increases to $\sim 9 \mathrm{MK}$ during the flare, while the hotter 

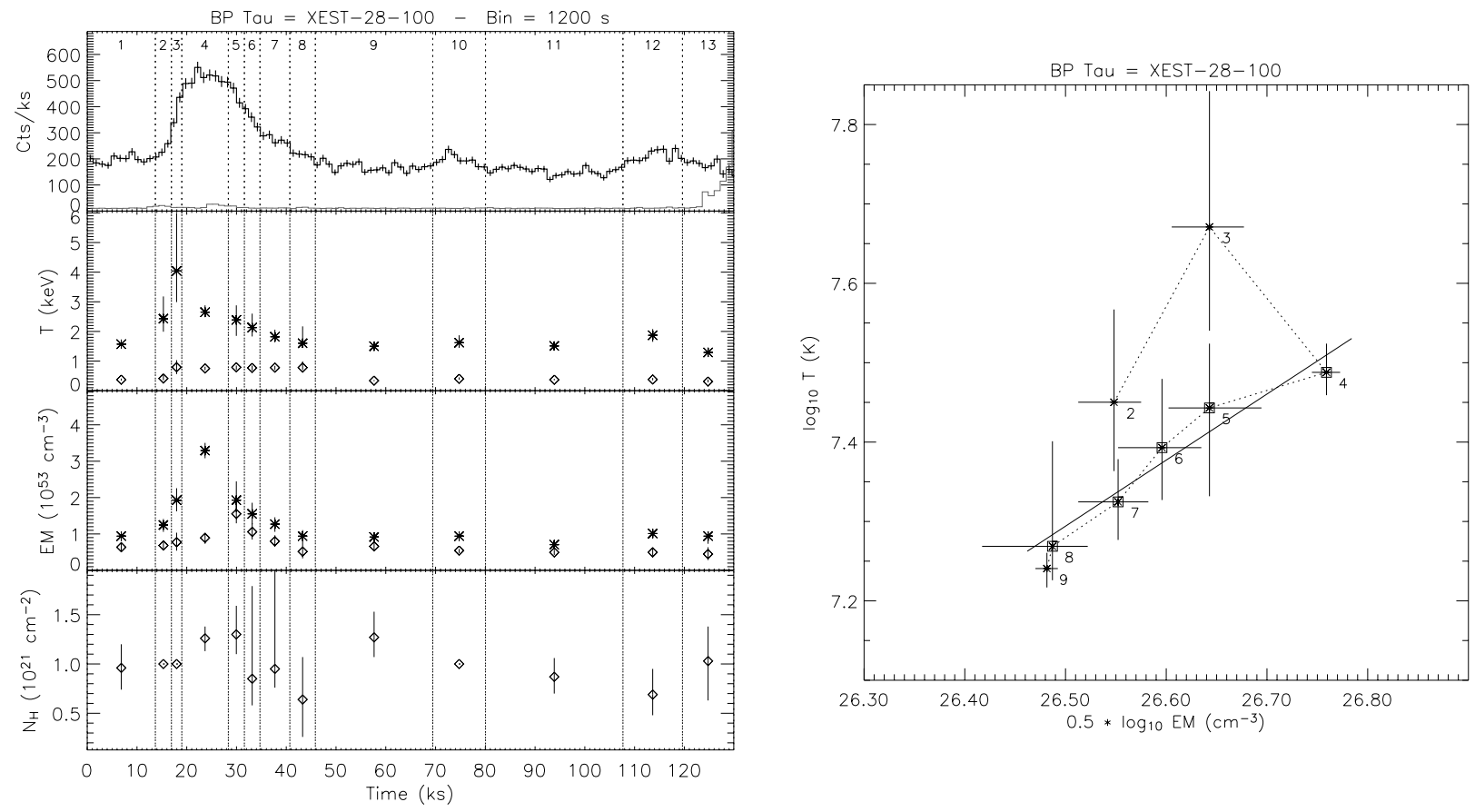

Fig. 3. Left: light curve and spectral fitting results for BP Tau (XEST-28-100). Panels are the same as in Fig. 1. Right: evolution of the best-fit temperature and EM of the hot component. See Fig. 2 for details. For the sake of clarity, we plot only the points from \#2 to \#9. The slope has been fitted between points \#4 and \#8.

component reaches $~ 50$ MK. Both EMs increase significantly during the flare.

The right panel of Fig. 3 shows the evolution of the flare temperature and EM. From Eq. (3), we derive a maximum loop temperature of $\sim 100 \mathrm{MK}$. The slope between points \#4 and \#8 is $\zeta=0.83 \pm 0.13$, indicating that residual heating is present during the decay. Using Eq. (2) we derive $L=1.2 \pm 0.1 \times 10^{11} \mathrm{~cm}$, comparable to the stellar radius. The estimated density at the flare peak is $n_{\mathrm{e}} \sim 3 \times 10^{10} \mathrm{~cm}^{-3}$, similar to the value found for HBC 427.

\subsubsection{JH 108 (XEST-17-066)}

JH 108 (XEST-17-066, WTTS, spectral type M1) shows a symmetrical flare, with a gradual increase of the count rate by a factor of $\sim 4$ in $\sim 8 \mathrm{ks}$, followed by a decay with similar duration; the decay e-folding time is $3.3 \mathrm{ks}$. This source has quiescent temperatures of 8 and $30 \mathrm{MK}$ and EM ratio of $\sim 1.5$. As before, the cool component remains nearly constant, while for the hotter component the temperature is higher during the rise phase, reaching $45 \mathrm{MK}$, and the EM peaks in the second time block, corresponding to the peak and decay phases. A rough estimate using Eq. (1) gives $L \lesssim 6 \times 10^{10} \mathrm{~cm}$, i.e., $\sim 0.7 R_{\star}$.

\subsubsection{V892 Tau (XEST-23-047/24-040)}

V892 Tau = Elias $1($ XEST-23-047/24-040) is a triple system composed of a Herbig Ae star, a low-mass companion of spectral type $\sim \mathrm{M} 2$ at 4.1" (Elias $1 \mathrm{NE}$, Leinert et al. 1997), and a recently-discovered close companion at $0.05^{\prime \prime}$ with a mass of 1.5-2 $M_{\odot}$ (Smith et al. 2005). For the first $95 \mathrm{ks}$ of the observation this source showed low-level variability, with temperatures in the range $T_{1}=5-10 \mathrm{MK}$ and $T_{2}=20-35 \mathrm{MK}$. Then the source underwent a strong flare, with a gradual rise lasting for $\sim 7 \mathrm{ks}$ and ending in a plateau, at a level a factor of $\sim 10$ higher than the pre-flare count rate, where the emission remained nearly constant for at least $10 \mathrm{ks}$, until the end of the observation. For the spectral analysis during the flare, the parameters of the cool component were kept fixed since they were not constrained, and the flare evolution was entirely dominated by the hot component. The plasma heating is strong in the first block of the rising phase, where the temperature increases from $\sim 25$ to $\sim 65 \mathrm{MK}$; then the temperature stays at a nearly constant level, within the errors, around $\sim 95-100 \mathrm{MK}$ in the following three blocks, and starts to decrease only when the count rate reaches the plateau in the last time block. The emission measure on the other hand continues to increase, reaching its maximum in the last block. The observed detailed evolution of the temperature and EM is a clear example of the flare heating process, in which, following the energy release, accelerated electrons precipitating into the chromosphere rapidly heat the plasma, and produce an evaporation of material that gradually fills the loop increasing the emission measure.

The long rise time suggests that a large coronal structure is involved in the flare. This flare has been studied previously by Giardino et al. (2004), who modeled the rise phase using detailed hydrodynamical simulations, finding a loop size of the order of $1 \times 10^{11} \mathrm{~cm}$, equal to $\sim 0.5 R_{\star}$, and that a magnetic field in excess of $500 \mathrm{G}$ is required to confine the flaring plasma. Based on the position of the X-ray source and on the comparison with a Chandra observation where the primary and Elias $1 \mathrm{NE}$ are resolved, Giardino et al. (2004) concluded that the flare occurred in the corona of the Herbig Ae star, which would imply the existence of a convective zone in the outer layers of the star, generally not expected in stars of this spectral type. However, the discovery of a lower-mass close companion suggests that the observed X-ray emission and the flare more likely originate from it rather than the Herbig star itself. This is supported by the high temperatures (20-30 MK) observed in this source and in other Herbig Ae/Be stars (Stelzer et al. 2006), similar to those commonly found for later-type PMS stars, but significantly higher 

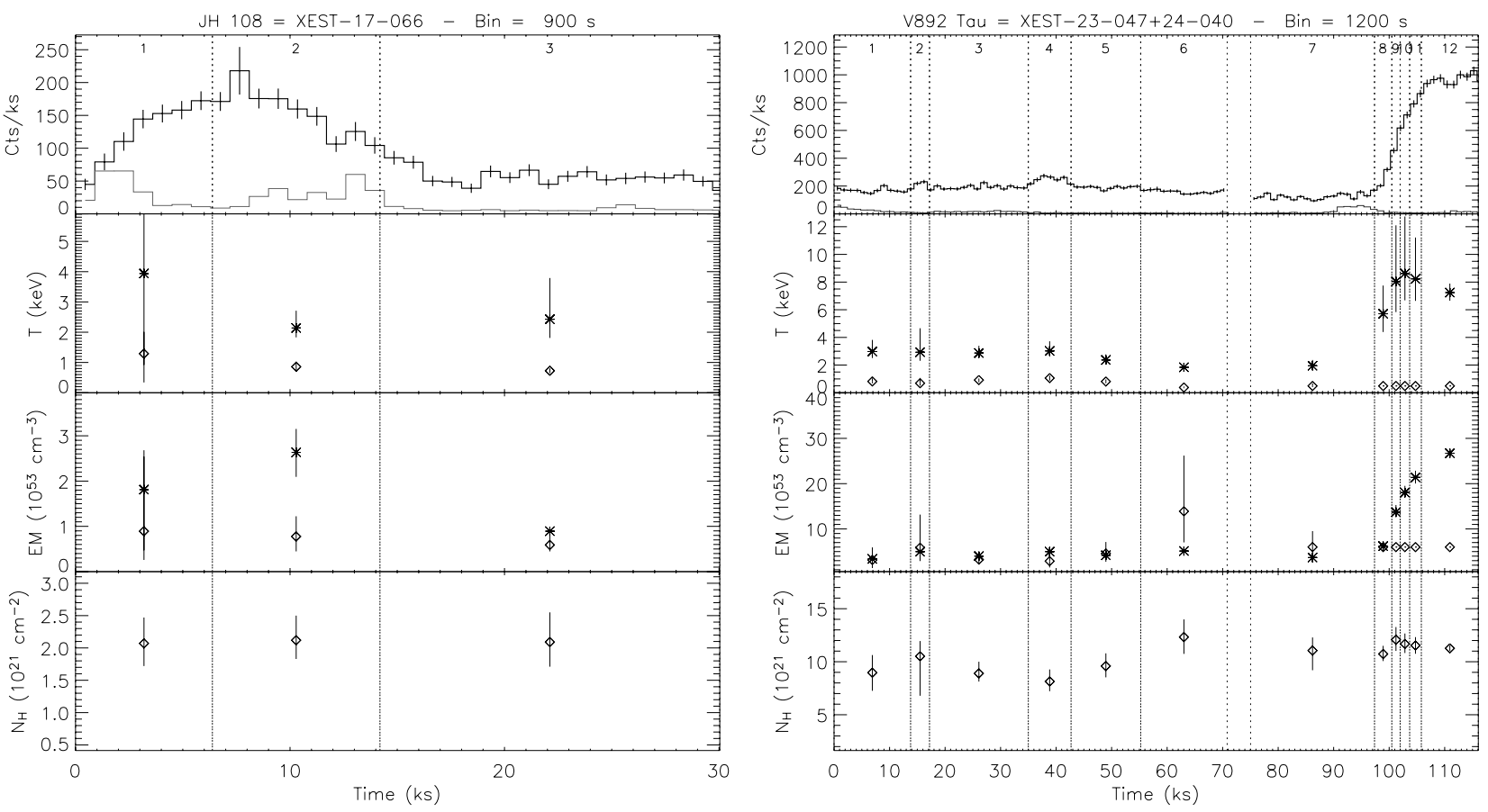

Fig. 4. Light curve and spectral fitting results for the TMC sources JH 108 (XEST-17-066) and V892 Tau (XEST-23-047/24-040) showing gradual rise flares. Panels are the same as in Fig. 1.

than those ( 5-6 MK) found in the Herbig stars HD 163296 and AB Aur (Swartz et al. 2005; Telleschi et al. 2007a).

\subsubsection{V410 Tau (XEST-23-032/24-028)}

The WTTS triple system V410 Tau (XEST-23-032/24-028) shows a very peculiar light curve with a nearly symmetrical triangular shape. The count rate rises gradually during the first 55 ks of observation until block \#8, where it reaches a peak a factor of $\sim 3$ higher than at the start of the observation. Then the emission decays until the last block; a second, weaker flare is superimposed on the decay $\sim 9 \mathrm{ks}$ before the end of the observation. Assuming that the first $18 \mathrm{ks}$ of the observation represent the quiescent level, we find that the rising phase has an e-folding time of $\sim 20 \pm 1 \mathrm{ks}$. After the peak the emission decays initially with an e-folding time of $8.1 \pm 0.4 \mathrm{ks}$ until the end of the XEST-23 exposure; at the beginning of the XEST-24 exposure the emission stays at a steady level for $23 \mathrm{ks}$ (block \#13), then the decay restarts, and continues until the end of the observation, interrupted only by the small flare.

During the observation, the temperature of the cool component does not vary significantly, and the flare evolution is determined by the variations of $T_{2}$ and of the EMs. In the rise phase the hot temperature initially increases from 20 to $30 \mathrm{MK}$, reaching a maximum in block \#4, then decreases again in the following two time intervals; a sudden increase to $\sim 45 \mathrm{MK}$ occurs in block \#7, just before the peak. The EMs of both plasma components increase during the entire rise phase, reaching their maximum at the flare peak, as commonly observed in solar and stellar flares. After the peak the temperature and the EMs decrease returning to the quiescent value. A new temperature increase is observed in block \#15, corresponding to the second small flare. The long rise time, combined with the slow change of the plasma temperature during the rise phase, might indicate that we are observing a rotationally modulated flare, or the superposition of a flaring event and an underlying modulated emission.
We also note that the best-fit column density increases by a factor of 4 during the flare, following a trend similar to that of the EMs. However, we found equally acceptable fits, although with slightly higher values (by at most 10\%) of the reduced $\chi^{2}$, by keeping $N_{\mathrm{H}}$ fixed to $1 \times 10^{20} \mathrm{~cm}^{-2}$, as found in the first time intervals, without significant changes in the time evolution of the other parameters. Since this star is a WTTS, which is not expected to possess circumstellar material that could justify changes in the intervening absorption, and that such low values of $N_{\mathrm{H}}$ are at the sensitivity limit of EPIC, we are not confident on the significance of the observed column density variations.

The right panel of Fig. 5 shows the evolution of the temperature and EM of the hot component during the flare. The slope during the decay, between blocks $\# 8$ and $\# 14$, is $\zeta=0.47 \pm 0.12$, indicating that the decay of the flaring structure is largely determined by the heating decay timescale. In this case the loop size is poorly constrained, given the large uncertainty on $\zeta$ : we obtain in fact $L \sim 4 \pm 3 \times 10^{10} \mathrm{~cm}$, or $0.1-0.4 R_{\star}$.

\subsection{Very long decays or increases of the count rate}

A group of six sources in our sample showed a monotonic decrease or increase of the count rate over the entire observation. One of them, XZ Tau, has a rising light curve, while for the other five sources (V830 Tau, HQ Tau, FS Tau, DH Tau, and L1551 51) a long-lasting decline of the count rate was observed. The light curves and best-fit parameters of this group of sources are shown in Figs. 6 and 7.

\subsubsection{XZ Tau (XEST-22-047)}

XZ Tau (XEST-22-047) is a binary star composed of two CTTS of spectral type M2 and M3.5. This source shows a long monotonic rise of the count rate by a factor of $\sim 4$ in $\sim 40 \mathrm{ks}$. An analysis of this observation has been reported by Favata et al. (2003) and Giardino et al. (2006). 

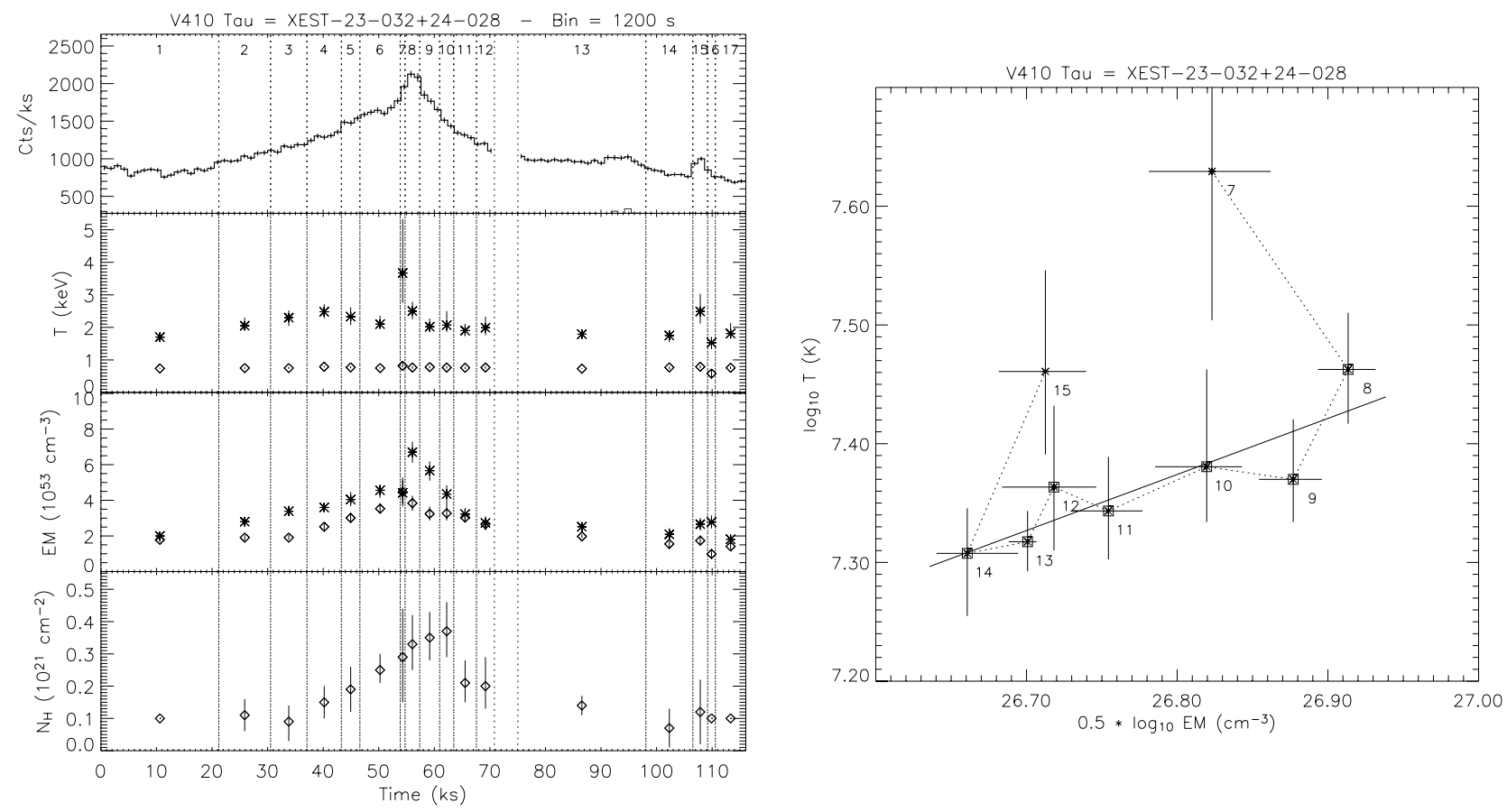

Fig. 5. Left: light curve and spectral fitting results for V410 Tau (XEST-23-032/24-028). Panels are the same as in Fig. 1. Right: evolution of the best-fit temperature and EM of the hot component. See Fig. 2 for details. For the sake of clarity, we plot only the points from \#7 to \#15. The slope has been fitted between points \#8 and \#14.

The quiescent emission at the beginning of the observation is rather hot, with temperatures of 8 and $40 \mathrm{MK}$ and $\mathrm{EM}_{2} / \mathrm{EM}_{1} \sim$ 0.7. The cool component does not vary significantly, except for an increase of the EM in the last block. The hotter temperature peaks at $\sim 70 \mathrm{MK}$ in blocks \#2 and 3, although the increase is not much significant due to the large errors, while the EM continues to increase as the count rate increases up to the last block. Our results are in agreement with those reported by Giardino et al. (2006). We note that the trend of $T_{2}$ and $\mathrm{EM}_{2}$ resembles that observed during the first part of the rising phase of V410 Tau, suggesting that we are observing a similar event.

\subsubsection{V830 Tau (XEST-04-016)}

V830 Tau (XEST-04-016, WTTS, spectral type K7) shows a long-lasting decay by a factor of 3 in $\sim 30 \mathrm{ks}$. The two temperatures remain nearly constant, within the errors, around $\sim 7$ and $20 \mathrm{MK}$ during the entire decay (apart from a possible increase in block \#3), and the observed decrease of the count rate is due only to the decrease of the two EMs with time. We note that also the average plasma temperature (weighted with the EMs) does not vary during the decay. It is possible that we are observing the final stage of a long-lasting flare, when the temperature has already returned to the pre-flare level while the EM is still changing. An alternative explanation might be rotational modulation: the relative amplitude of the count rate variation around the average count rate is $\sim 60 \%$, compatible with the range of $20-70 \%$ found in the Orion Nebula Cluster (Flaccomio et al. 2005). However, given the high amplitude of the variation, and the fact that the $30 \mathrm{ks}$ exposure covers only one tenth of the star's rotational period ( $P_{\text {rot }}=2.75 \mathrm{~d}$, see Table 10 in Güdel et al. 2007a), we believe that this interpretation is unlikely, although we cannot draw definitive conclusions from the available data.

\subsubsection{HQ Tau (XEST-09-026)}

HQ Tau (XEST-09-026) is a close binary WTTS. Its light curve shows a long-lasting decay, with a decrease of the count rate by a factor of 2 in $30 \mathrm{ks}$. The spectral analysis shows a significant decrease of the hotter temperature, from 34 to $20 \mathrm{MK}$, and of both EMs, while the temperature of the cool component remains constant. This behavior suggests that we are observing the end of the decay of a long-duration flare. The e-folding timescale of the observed decay is $\sim 45 \mathrm{ks}$. From the decay of the temperature and EM of the hottest component we find a high slope $\zeta \sim 2$, compatible with a freely-decaying loop. Using Eq. (1) we obtain $L \gtrsim 7 \times 10^{11} \mathrm{~cm}$. Since the stellar radius of this star is not known, we cannot determine whether this represents a compact or a large loop.

\subsubsection{FS Tau (XEST-11-057)}

FS Tau (XEST-11-057) is a binary composed of two CTTS of spectral type M0 and M3.5. Its light curve decreases by a factor of $\sim 6$ during the first $20 \mathrm{ks}$, with an e-folding timescale of $15 \pm$ $2 \mathrm{ks}$, and then remains at a low quiescent level for the remaining $20 \mathrm{ks}$. In this case the spectra are well described by just one temperature, the low-temperature component being unconstrained due to the high absorption $\left(N_{\mathrm{H}} \sim 1-1.6 \times 10^{22} \mathrm{~cm}^{-2}\right)$. The temperature does not change significantly, decreasing from $40 \mathrm{MK}$ to $35 \mathrm{MK}$ from the beginning to the end of the observation. On the other hand the EM decreases significantly; a small but significant decrease of the absorption is also observed. In this case, it is not possible to apply the Reale et al. (1997) method, since the rate of decrease of the temperature is too low $(\zeta=0.2)$. Applying the formula for a freely-decaying loop we estimate an upper limit to the loop length of $L \sim 2.7 \times 10^{11} \mathrm{~cm}\left(\sim 4 R_{\star}\right)$. Note that, although the peak temperature of the flare is likely higher and therefore the above upper limit is underestimated, we expect the 

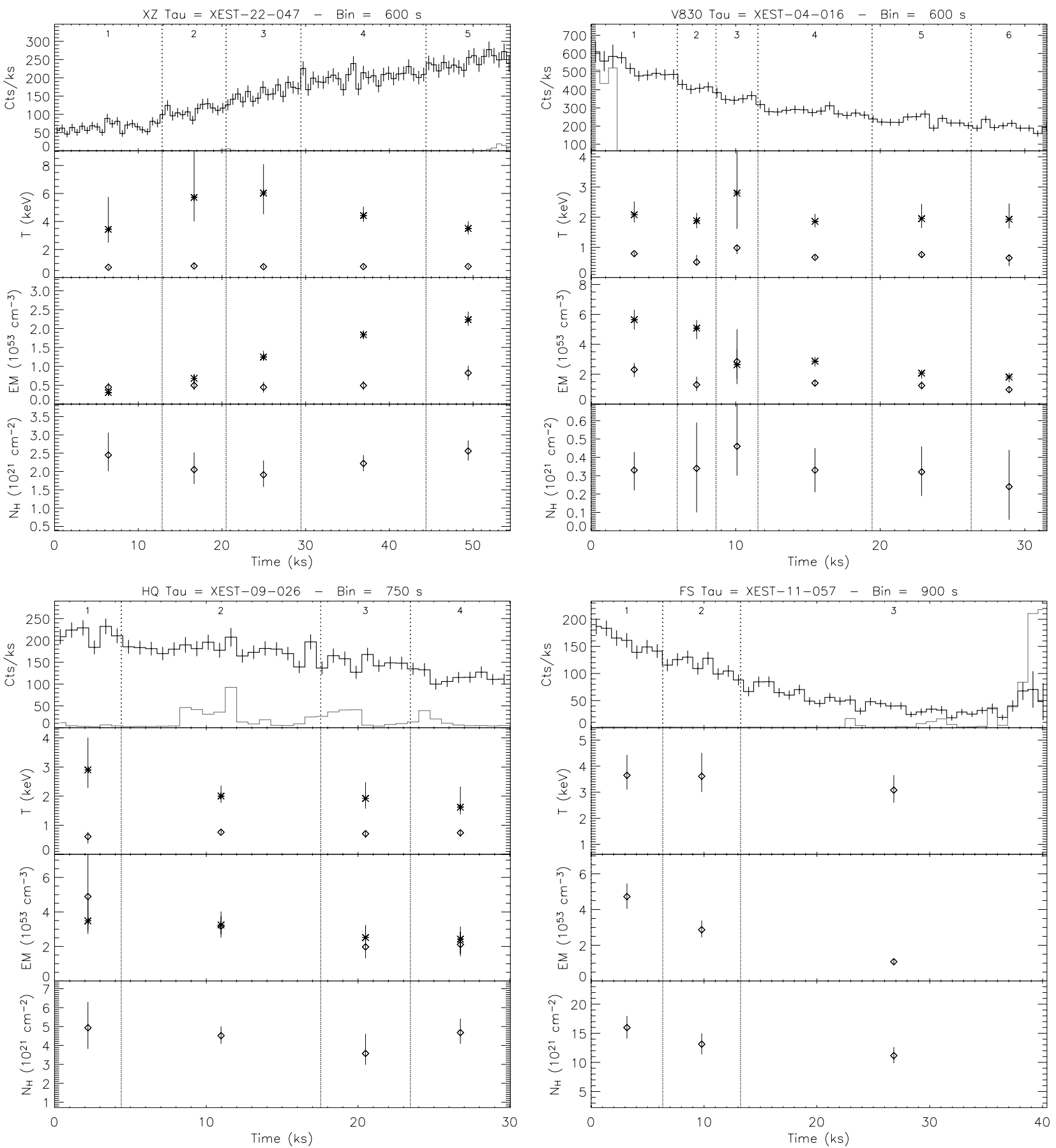

Fig. 6. Light curve and spectral fitting results for TMC sources showing a long-lasting rise $(\mathrm{XZ}$ Tau $=$ XEST-22-047) or decay $($ V830 Tau $=$ XEST-04-016, HQ Tau = XEST-09-026, FS Tau = XEST-11-057). Panels are the same as in Fig. 1.

true loop size to be much smaller because of the presence of strong sustained heating, as indicated by the shallow slope $\zeta$.

\subsubsection{DH Tau (XEST-15-040)}

DH Tau (XEST-15-040) is a binary CTTS with a separation of 2.3", unresolved by XMM-Newton. It shows a monotonic decay by a factor of $\sim 3$ over the $30 \mathrm{ks}$ exposure. As for V830 Tau, the two temperatures are steady during the decay, at $\sim 9$ and $\sim 25 \mathrm{MK}$, while the two EMs decrease. In this case, contrary to V830 Tau, the ratio $\mathrm{EM}_{2} / \mathrm{EM}_{1}$ also decreases, implying that, as time proceeds, less and less hot material contributes to the emission. This suggests that we are observing the end of a longlasting flaring event. As for the previous source, very strong sustained heating is present $(\zeta=0.2)$, preventing the use of the Reale et al. (1997) method, and we estimate $L<4.5 \times$ $10^{11} \mathrm{~cm} \sim 3.5 R_{\star}$.

\subsubsection{L1551 51 (XEST-22-089)}

L1551 51 (XEST-22-089, WTTS) also shows a decay by a factor of 2 during the first $30 \mathrm{ks}$, then stays at a constant level for the remaining $20 \mathrm{ks}$ of observation. Also for this source the temperatures do not vary, and the observed variation is due only to the 

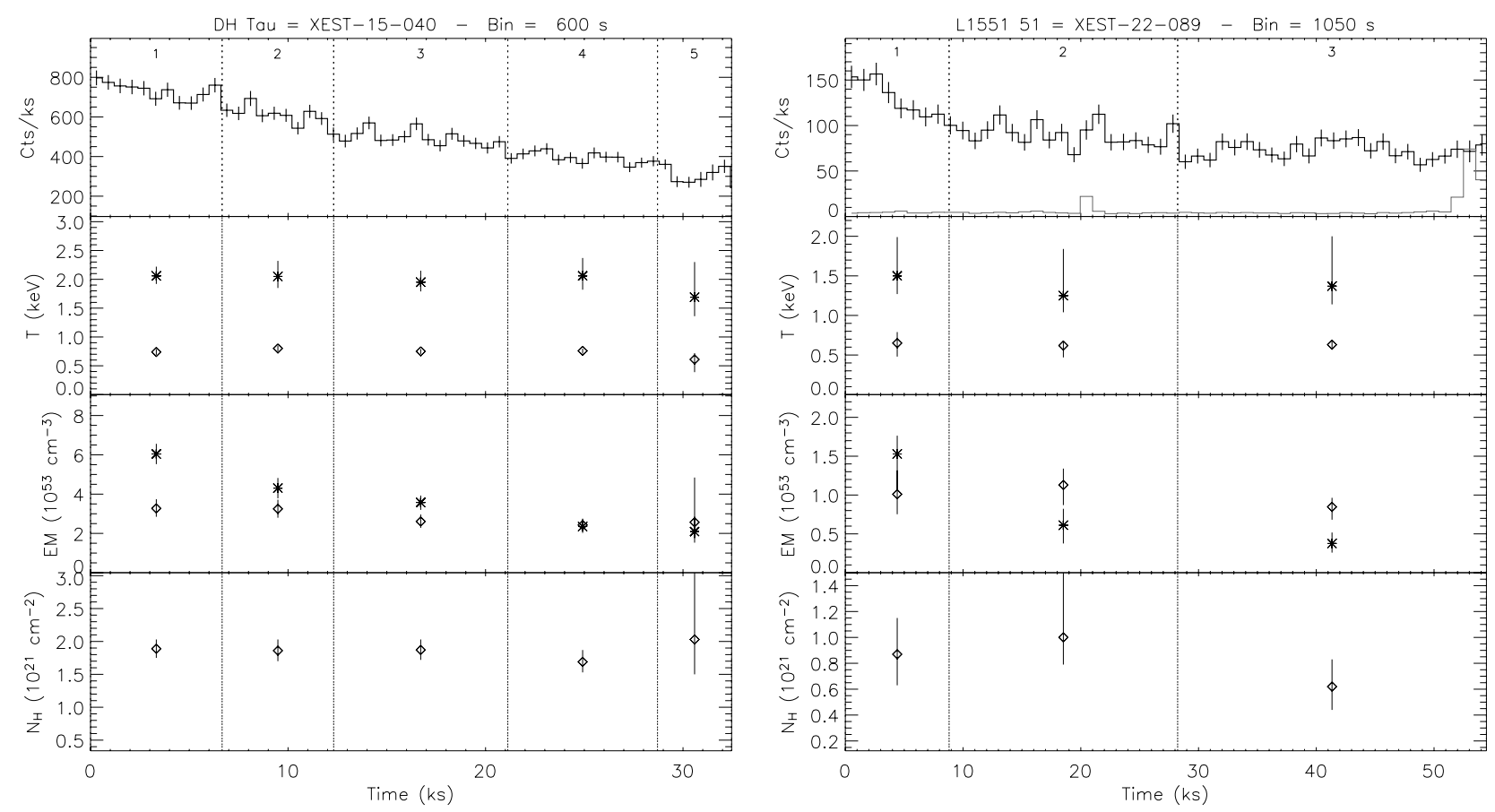

Fig. 7. Same as Fig. 6 for DH Tau (XEST-15-040) and L1551 51 (XEST-22-089).

changing EMs. As for DH Tau, the EM ratio also decreases, implying a decrease of the average plasma temperature, likely due to a flare decay. This source also shows very strong sustained heating $(\zeta=0.16)$. From the decay e-folding time of $9.5 \mathrm{ks}$, we estimate $L \lesssim 1 \times 10^{11} \mathrm{~cm}$, comparable to the stellar radius.

\subsection{Other types of variability}

The remaining three XEST sources identified with TMC members show significant variability in the form of slow modulation (DN Tau and HD 283572, Fig. 8) or complex flare-like variability (Hubble 4, Fig. 9).

\subsubsection{DN Tau (XEST-12-040)}

DN Tau (XEST-12-040, CTTS, spectral type M0) shows a modulated light curve with a higher count rate at the beginning and the end of the exposure and a minimum in the second time block; the amplitude of the modulation with respect to the mean level is $\sim 15 \%$. The plasma parameters do not show significant variations, being consistent within the errors, although there is a tendency for higher $T_{2}$ and $\mathrm{EM}_{1}$ in the first and last block.

\subsubsection{HD 283572 (XEST-21-039)}

HD 283572 (XEST-21-039) is a G5 WTTS with $P_{\text {rot }}=1.55 \mathrm{~d}$; the present observation, with an exposure time of $\sim 45 \mathrm{ks}$, therefore covers about one third of the rotational period. The light curve shows a clear modulation, with two characteristic levels of emission. During the first $20 \mathrm{ks}$ the count rate increases slightly, then in block \#4 it decreases reaching a level a factor of $\sim 1.3$ lower, which is maintained until the end of the observation. The two temperatures do not vary significantly, with the cooler one staying around 8-9 MK, and the hotter one varying between 20 and $25 \mathrm{MK}$ with a trend that closely follows that of the light curve. On the other hand, the two EMs show significant variations, in particular in the first part of the observation, where the cool EM decreases and the hot one increases, leading to an increase in their ratio and therefore in the relative contribution of the hotter plasma to the emission. These characteristics suggest that the observed variability is likely due to the appearing and disappearing, due to the stellar rotation, of active regions containing hotter plasma with respect to the rest of the corona.

\subsubsection{Hubble 4 (XEST-23-056/24-047)}

The light curve of Hubble 4 (XEST-23-056/24-047, WTTS, spectral type K7) shows significant low-level flare-like variability superimposed on a slow modulation. The average count rate shows a very slow decrease during the first $50 \mathrm{ks}$, then it increases by a factor of $\sim 1.5$ at the beginning of block \#4, and decays again slowly until the end of the observation. The two temperatures do not vary significantly, having average values of $\sim 9 \mathrm{MK}$ and $\sim 20 \mathrm{MK}$, with the exception of the last block where the hot component is slightly higher. As in other sources, the variations of the two EMs are more significant, showing a modulation with time of both values, but keeping their ratio nearly constant, between 1 and 1.2. The observed slow variation of the mean count rate might be due to rotational modulation of the emission from active regions rotating in and out of view.

\section{Sources not associated with known TMC members}

In addition to the TMC members, we have studied three additional XEST sources (XEST-05-031 = HD 283810, XEST$16-031=2$ MASS J04195676+2714488, and XEST-22-024 = HD 285845) that are likely non-members of the TMC, but that are strong $\mathrm{X}$-ray sources and show significant variability. Their light curves and spectral parameters are shown in Figs. 10 and 11. 

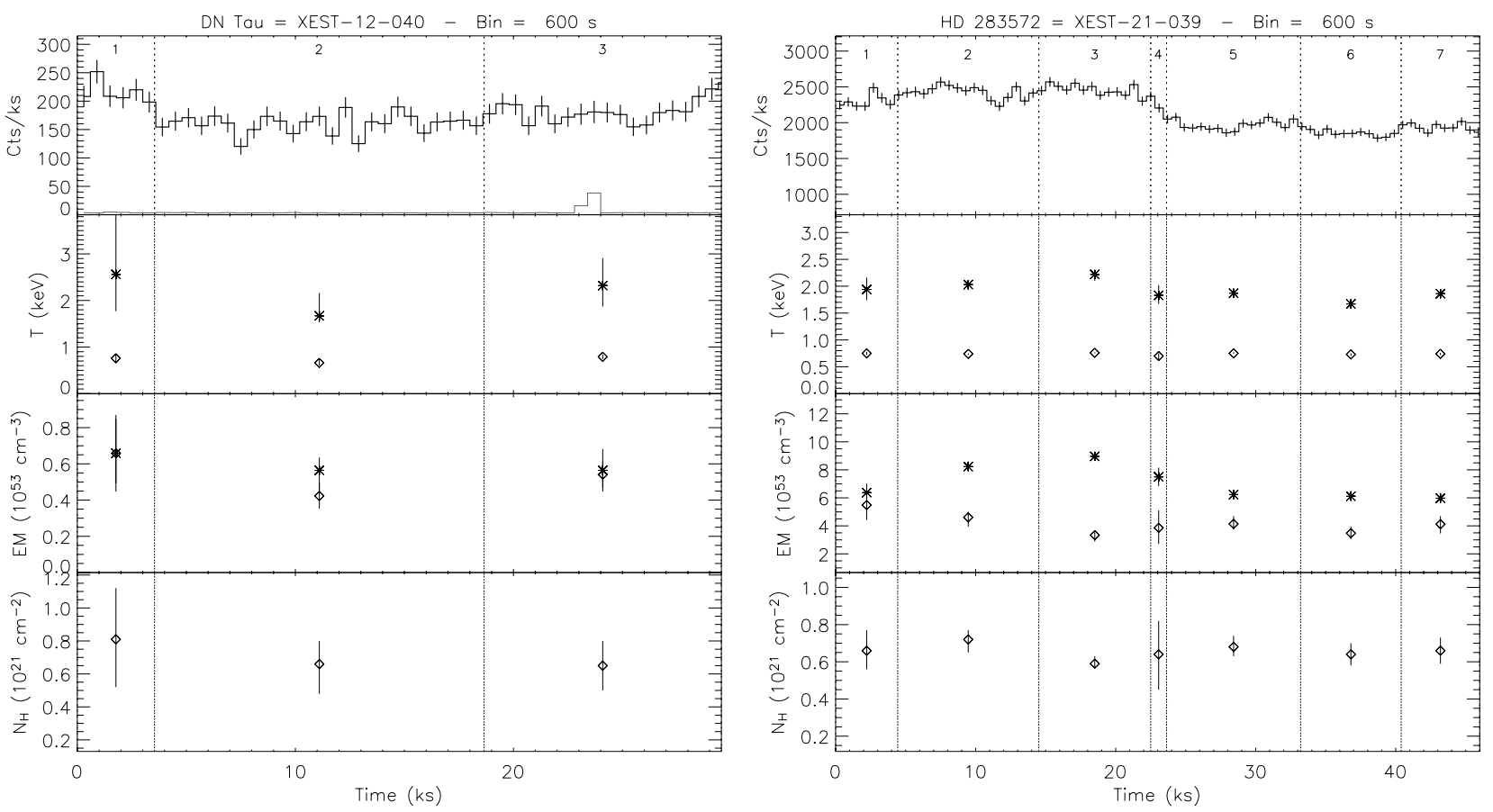

Fig. 8. Light curve and spectral fitting results for the TMC sources DN Tau (XEST-12-040) and HD 283572 (XEST-21-039), showing a slow modulation. Panels are the same as in Fig. 1.

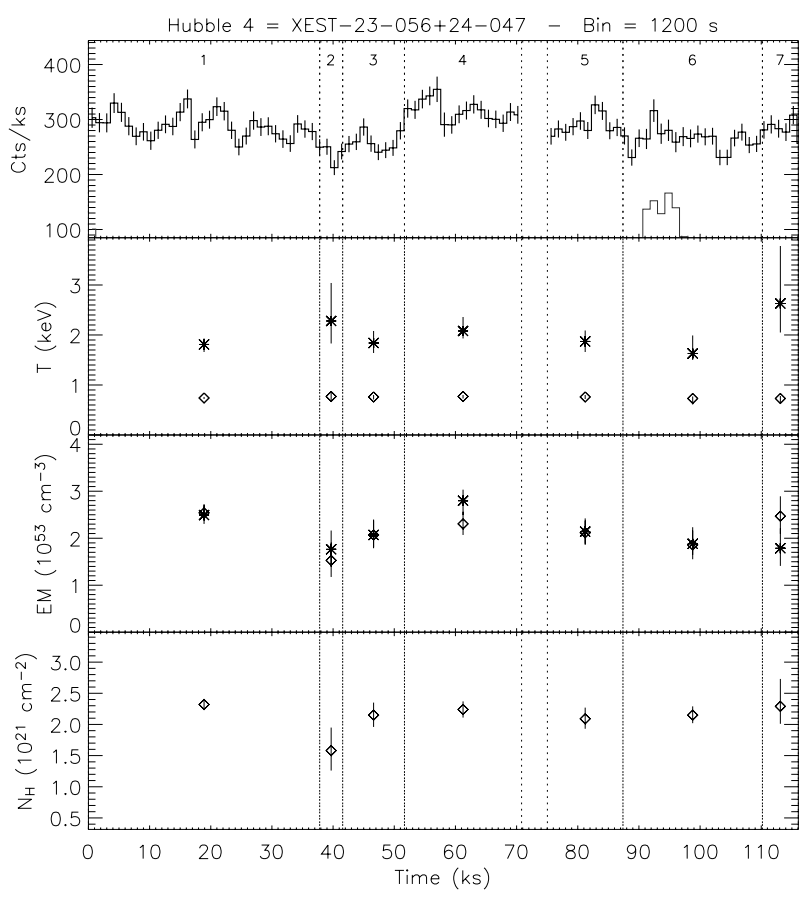

Fig. 9. Light curve and spectral fitting results for the TMC source Hubble 4 (XEST-23-056/24-047) showing a complex variability. Panels are the same as in Fig. 1.

\subsection{HD 283810 (XEST-05-031)}

XEST-05-031 is identified with HD 283810, a K5V star with $\mathrm{H} \alpha$ emission, which is probably an older foreground star since it has radial velocity inconsistent with TMC membership and does not show significant Li absorption (Herbig et al. 1986); assuming a main-sequence object, its photometry $(V=10.74, B-V=$ 1.03 ) locates it at a distance of $\sim 60 \mathrm{pc}$. Its light curve shows a flare at the end of the observation, with an increase of the count rate by a factor of 2.5 and an e-folding decay time of $\sim 2.4 \mathrm{ks}$. The hotter temperature rises from $\sim 9 \mathrm{MK}$ during quiescence to $18 \mathrm{MK}$ in block \#3 and reaches a maximum value of $26 \mathrm{MK}$ in block \#4, after the flare peak. Both EMs increase significantly at the flare peak. Given the irregular trend of the temperature and EM, we cannot apply the Reale et al. (1997) method; using the formula for a freely decaying loop and the observed temperature in block \#3, we derive a loop semi-length of $L<3 \times 10^{10} \mathrm{~cm}$, which is comparable to the stellar radius for a K5 main-sequence star (Siess et al. 2000).

\subsection{HD 285845 (XEST-22-024)}

XEST-22-024 is identified with HD 285845, which is a foreground binary system at a distance of $90 \mathrm{pc}$; the absence of the $\mathrm{Li}$ absorption line at $6708 \AA$ indicates that the system is not composed of PMS stars (Walter et al. 1988; Favata et al. 2003). The mean properties of the X-ray spectrum have been discussed by Favata et al. (2003), who reported a spectral fitting with a $2-\mathrm{T}$ model characterized by high $\mathrm{Ca}$ and $\mathrm{Ne}$ abundances with respect to a Fe abundance around $0.26 Z_{\odot}$.

The light curve of this source shows a complex variability. A flare occurred at the beginning of the exposure, with a gradual rise lasting $\sim 5 \mathrm{ks}$, followed by a decay on which several minor impulses are superimposed. The initial decay just after the peak has an e-folding time of $\sim 2 \mathrm{ks}$. The emission reaches a steady level $\sim 30 \mathrm{ks}$ after the peak. As observed in other sources, the cool temperature does not vary significantly during the flare evolution. The hotter temperature peaks in block \#2, during the rise phase, at $40 \mathrm{MK}$, and remains steady at a level of $\sim 30-35 \mathrm{MK}$ up to block \#7, when it decreases to $\sim 15-25 \mathrm{MK}$, remaining at this level until the end of the observation. The observed light curve variations are mostly due to the EMs of both components, which vary significantly throughout the observation. Given the complexity of the light curve, it is not possible to derive the loop 

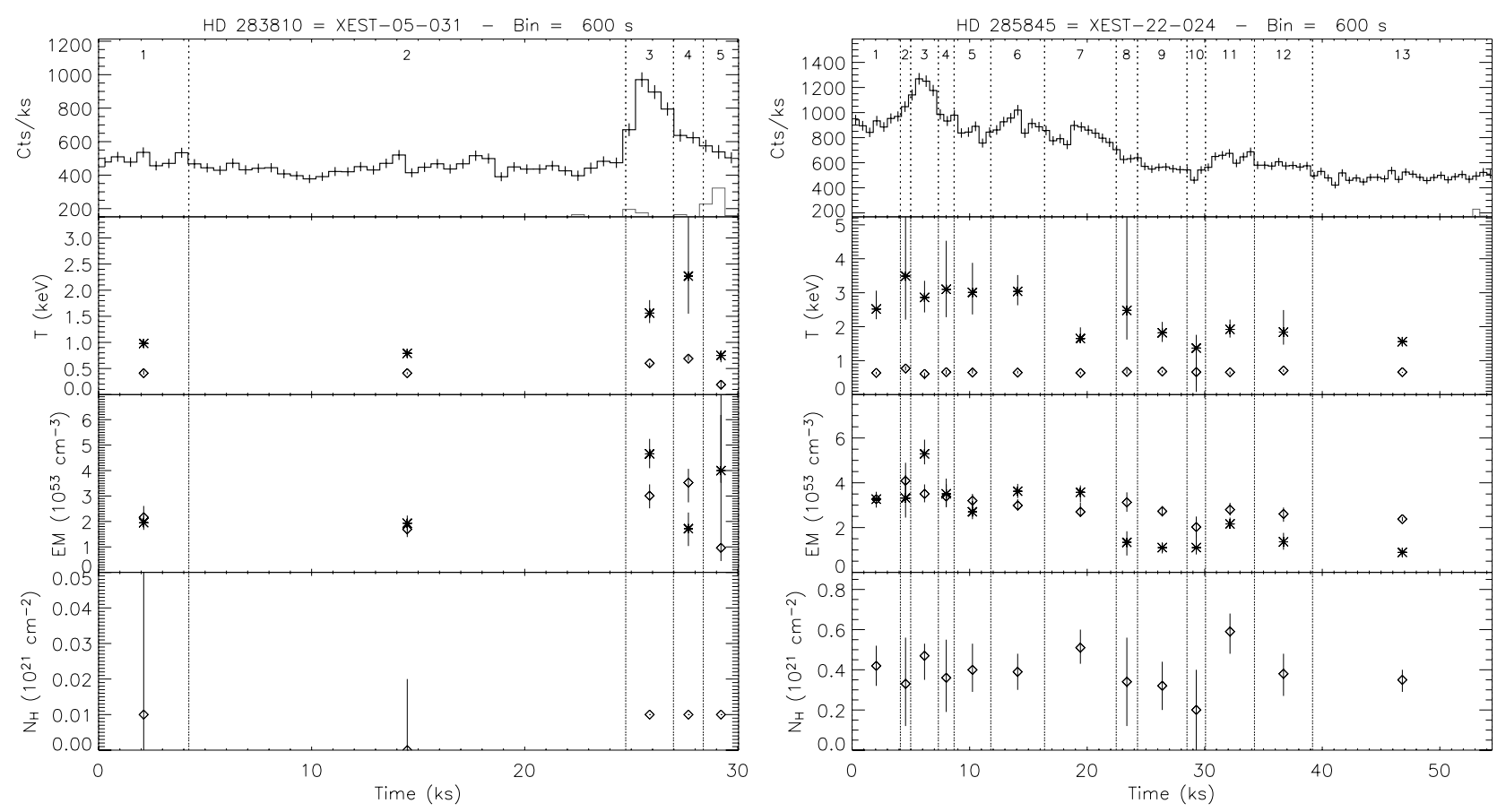

Fig. 10. Light curve and spectral fitting results for the XEST sources HD 283810 (XEST-05-031) and HD 285845 (XEST-22-024), not associated with the TMC. Panels are the same as in Fig. 1.

size by fitting the $T$ vs. EM decay. We can make a rough estimate assuming the initial decay time of $2 \mathrm{ks}$ and Eq. (1), obtaining $L \sim 3 \times 10^{10} \mathrm{~cm}$. Assuming a G6 main-sequence star, this corresponds to $\sim 0.4 R_{\star}$ (Siess et al. 2000).

\subsection{MASS J04195676+2714488 (XEST-16-031)}

Source XEST-16-031 has an IR counterpart in the 2MASS catalogue, 2MASS J04195676+2714488, located at $\sim 0.2^{\prime \prime}$ and with magnitudes $J=12.38, H=11.80$, and $K=11.54 \mathrm{mag}$; another fainter 2MASS source is present at $4.6^{\prime \prime}$, however the X-ray source is most likely associated with the former one, given the accuracy of the XEST positions (see Güdel et al. 2007a; Scelsi et al. 2007). 2MASS J04195676+2714488 has photometry inconsistent with TMC membership, being located on the main sequence in the color-magnitude diagrams for the distance of the TMC (Scelsi et al. 2007), and is therefore likely to be a field late-type star. The uncertainties in the photometry allow a distance between 80 and 190 pc for a main sequence star.

During the first $23 \mathrm{ks}$, the source was in a quiescent state with very low emission. Unfortunately in this part of the observation (blocks \#1 to 3 ) the background was very high and dominating the observed count rate (see Fig. 11), preventing the possibility of performing spectral analysis. In block \#4, $24 \mathrm{ks}$ after the start of the observation, a flare occurred, followed by a second much stronger flare $7 \mathrm{ks}$ later. The first flare has a peak count rate a factor of $\sim 5$ higher than the quiescent level, while the second flare increases the count rate by more than one order of magnitude. The two flares have very similar decay times, with e-folding times of $\sim 1.6$ and $1.5 \mathrm{ks}$, respectively. This suggests that they may have occurred in loops of comparable size.

The absorption is very low, consistent with a column density $N_{\mathrm{H}}=1 \times 10^{20} \mathrm{~cm}^{-2}$, and to better constrain the fit parameters it was kept fixed to this value. The spectrum was well fitted with only one temperature component. Since the emission during the quiescent phase is negligible with respect to the flare phase, we assume that the 1-T model is essentially representing the flaring plasma during the time blocks from \#4 to \#14.

The right panel of Fig. 11 shows the evolution of the temperature and the EM during the observation. In block \#4, corresponding to the peak of the first, small flare, the plasma temperature is $22 \mathrm{MK}$; the temperature and EM decrease in block \#5 during the flare decay, and remain steady in the following time interval. In block \#7, at the start of the rise of the second flare, the temperature increases to $\sim 42 \mathrm{MK}$; a second re-heating is observed in block \#9, corresponding to the flare peak, where the EM reaches its maximum value. The flare cooling phase from point \#10 to point \#14 proceeds with a slope $\zeta=0.42 \pm 0.07$, which indicates the presence of significant sustained heating after the initial ignition. It is worth noting that the slope between points \#4 and \#5, relative to the decay of the first small flare, has a very similar value of 0.37 . Using Eq. (2) we derive $L \sim 1 \times$ $10^{9} \mathrm{~cm}$ for the first flare, and $L \sim 5 \pm 4 \times 10^{9} \mathrm{~cm}$ for the second flare. These values are significantly smaller than those estimated for the other sources. Although the precise nature of this source cannot be assessed here, given the very low absorption we suggest that it could be an older M-type star located just in front of the cloud and showing flare events analogous to those observed on the Sun and active late-type stars.

\section{Discussion and conclusions}

In this paper we have studied a sample of 19 bright variable $\mathrm{X}$-ray sources detected in the XEST survey. Our sample includes 16 known TMC members (10 WTTS, 5 CTTS, and a Herbig Ae star) plus three additional sources unrelated to the cloud but showing flaring events. The studied sources show different types of variability, in the form of flares, either with fast rise and slower decay or with symmetrical shapes, continuous rise or decays, slow modulation possibly due to rotation, and complex variability, with flare-like events superimposed on a slow modulation or decay. Using detailed time-resolved 

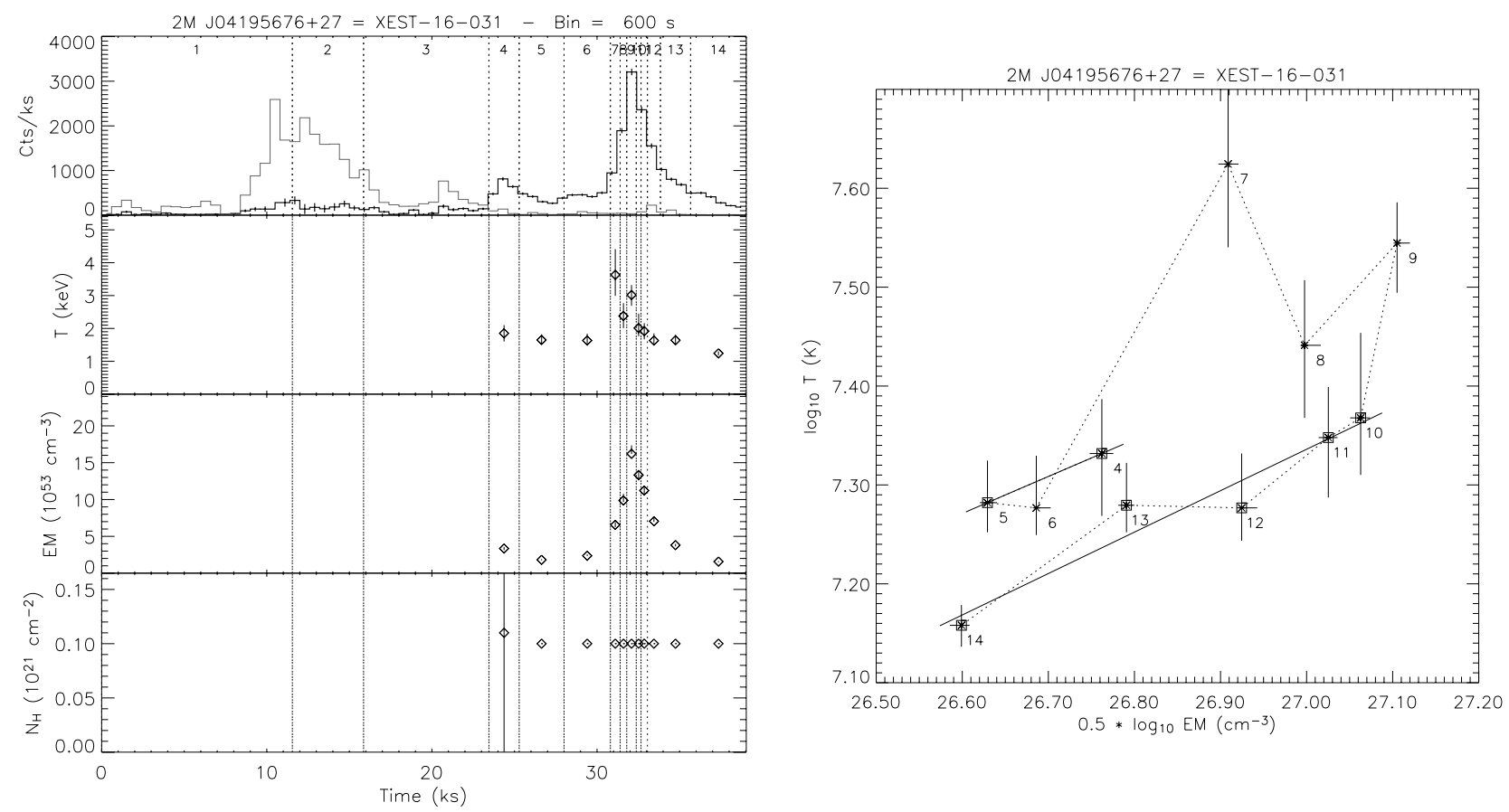

Fig. 11. Left: light curve and spectral fitting results for XEST-16-031. Panels are the same as in Fig. 1. Right: evolution of the best-fit temperature and EM of the hot component. See Fig. 2 for details. The slope has been fitted between points \#10 and \#14. The slope during the decay of the first small flare (points \#4 and \#5) is also indicated.

Table 3. Summary of the parameters derived for the XEST sources in quiescence and during flares.

\begin{tabular}{|c|c|c|c|c|c|c|c|c|c|}
\hline XEST ID & Optical ID & $\begin{array}{c}T_{\mathrm{q}}^{a} \\
(\mathrm{MK})\end{array}$ & $\begin{array}{c}L_{\mathrm{Xq}}^{a} \\
\left(10^{30} \mathrm{erg} \mathrm{s}^{-1}\right)\end{array}$ & $\begin{array}{r}T_{\text {peak }}^{b} \\
(\mathrm{MK})\end{array}$ & $\begin{array}{c}L_{\text {Xpeak }}^{b} \\
\left(10^{30} \mathrm{erg} \mathrm{s}^{-1}\right)\end{array}$ & $\begin{array}{l}\tau_{\text {rise }}^{c} \\
(\mathrm{ks})\end{array}$ & $\begin{array}{l}\tau_{\mathrm{dec}}^{c} \\
(\mathrm{ks})\end{array}$ & $\begin{array}{c}L \\
\left(10^{11} \mathrm{~cm}\right)\end{array}$ & $L / R_{\star}$ \\
\hline 04-016 & V830 Tau & 22 & 2.8 & $\geq 33$ & $\geq 8.5$ & $\cdots$ & 11.4 & $\cdots$ & $\cdots$ \\
\hline 09-026 & HQ Tau & 19 & 4.6 & $\geq 34$ & $\geq 9.1$ & $\ldots$ & 45.2 & $\gtrsim 7.1$ & $\cdots$ \\
\hline $11-057$ & FS Tau & 36 & 1.4 & $\geq 42$ & $\geq 6.3$ & $\ldots$ & 15.2 & $\lesssim 2.7$ & 4.1 \\
\hline $12-040$ & DN Tau & $19-27$ & $1.0-1.4$ & $\cdots$ & $\cdots$ & $\cdots$ & $\ldots$ & $\ldots$ & $\cdots$ \\
\hline $15-040$ & DH Tau & 20 & 4.7 & $\geq 24$ & $\geq 9.8$ & $\cdots$ & 34.2 & $\lesssim 4.5$ & 3.5 \\
\hline $17-066$ & JH 108 & 28 & 1.5 & 46 & 3.7 & 3.8 & 3.3 & $\lesssim 0.6$ & 0.7 \\
\hline 21-039 & HD 283572 & $19-26$ & $10-14$ & $\cdots$ & $\cdots$ & $\cdots$ & $\cdots$ & $\cdots$ & $\cdots$ \\
\hline $22-047$ & XZ Tau & 40 & 0.9 & 70 & 3.8 & 35.3 & $\cdots$ & $\cdots$ & $\cdots$ \\
\hline $22-089$ & L1551 51 & 16 & 1.4 & $\geq 17$ & $\geq 2.5$ & $\ldots$ & 9.5 & $\lesssim 1.1$ & 1.1 \\
\hline $23-032 / 24-028$ & V410 Tau & 20 & 3.8 & 43 & 11.7 & 20.9 & 8.1 & $0.1-0.7$ & $0.1-0.4$ \\
\hline $23-047 / 24-040$ & V892 Tau & 23 & 9.5 & 100 & 46.8 & 3.4 & $\cdots$ & $1.0^{d}$ & 0.5 \\
\hline $23-050 / 24-042$ & V410 X7 & 24 & 0.7 & $\geq 63$ & $\geq 4.9$ & 1.5 & 2.1 & $\geq 0.5$ & 0.4 \\
\hline $23-056 / 24-047$ & Hubble 4 & $19-30$ & $3.5-5.4$ & $\ldots$ & $\ldots$ & $\cdots$ & $\ldots$ & $\cdots$ & $\cdots$ \\
\hline $23-074 / 24-061$ & V819 Tau & 14 & 2.0 & 19 & 3.8 & 1.3 & 10.1 & 0.7 & 0.5 \\
\hline $26-072$ & HBC 427 & 25 & 3.2 & 72 & 15.8 & 3.2 & 20.2 & 2.4 & 1.9 \\
\hline $28-100$ & BP Tau & 18 & 1.5 & 47 & 4.9 & 2.6 & 10.4 & 1.2 & 0.9 \\
\hline $05-031$ & HD 283810 & 10 & $1.5^{e}$ & 26 & $3.2^{e}$ & 0.5 & 2.4 & 0.3 & $\cdots$ \\
\hline $16-031$ & $\mathrm{~J} 04195676+27$ & $\cdots$ & $\cdots$ & 21 & 3.5 & 0.6 & 1.6 & 0.01 & $\cdots$ \\
\hline$"$ & $"$ & $\cdots$ & $\cdots$ & 42 & 20.0 & 0.8 & 1.5 & $0.01-0.09$ & $\cdots$ \\
\hline $22-024$ & HD 285845 & 18 & $2.1^{f}$ & 40 & $6.5^{f}$ & $\cdots$ & $\ldots$ & $\ldots$ & $\cdots$ \\
\hline
\end{tabular}

${ }^{a}$ Temperature of the hot component and luminosity in the quiescent (or lowest count rate) intervals; a range is given for sources with slow modulation or complex variability. ${ }^{b}$ Peak temperature and luminosity for sources showing flares or prolonged rise/decays. ${ }^{c}$ Rise and decay e-folding timescales. ${ }^{d}$ From Giardino et al. (2004). ${ }^{e}$ Computed for $d=60$ pc. ${ }^{f}$ Computed for $d=90$ pc.

spectroscopy we have investigated the changes of the parameters of the emitting plasma (temperatures and emission measures) and of the hydrogen column density, and, in the case of flares, we have derived information on the size of the involved coronal structures. The main parameters derived for the studied sources are summarized in Table 3.

The quiescent emission has typical temperatures $T_{1} \sim$ 4-10 $\mathrm{MK}$ and $T_{2} \sim 15-35 \mathrm{MK}$, with $\mathrm{EM}_{2} / \mathrm{EM}_{1} \sim 0.7-2$, consistent with the values found in other studies of young PMS stars (e.g., Feigelson \& Montmerle 1999; Wolk et al. 2005). No significant difference is evident in our small sample between the spectral characteristics of CTTS and WTTS: both the ranges of the parameters and their median values are similar for the two classes. The Herbig Ae star V892 Tau has spectral characteristics very similar to those found for TTS stars, in agreement with other studies 
(Hamaguchi et al. 2005; Stelzer et al. 2006), supporting the suggestion that the emission might come from its cool, unresolved close companion rather than from the Herbig Ae star itself. We find that in most sources the cool plasma component does not vary significantly, even during flares, while the observed time evolution can be totally ascribed to variations in the hot component. Similar results have been obtained for the Orion PMS stars studied in the COUP survey (Wolk et al. 2005), as well as for older active stars (e.g., Audard et al. 2001).

Nine of the studied sources (including two TMC nonmembers) show evident flaring activity. The flares observed on TMC members have a total duration between $\sim 20$ and $70 \mathrm{ks}$ ( $\sim 5-20 \mathrm{~h}$ ), with e-folding rise timescales of $\sim 1-4 \mathrm{ks}$, except for the peculiar flare on V410 Tau with $\tau_{\text {rise }} \sim 20 \mathrm{ks}$, and decay timescales of $\sim 2-20 \mathrm{ks}$. An additional 5 sources show gradual decays over 30-50 ks, which might represent the decay of longlasting flares, as suggested by the decreasing plasma temperature and emission measure, and one source shows a prolonged rise (with e-folding timescale of $\sim 35 \mathrm{ks}$ ) with spectral characteristics similar to those observed during the long rise phase of the atypical flare on V410 Tau. Unfortunately, the typical 30-40 ks exposure times of the XEST survey introduce a bias against the detection of long-duration flares in our observations. Indeed, we detected flares with a total duration of $\sim 50-70 \mathrm{ks}$ in the archival fields having exposure times of $\sim 100 \mathrm{ks}$. It is conceivable that the sources with gradual decays were undergoing flares of similar duration or even longer, such as found in the COUP survey, where events lasting up to 3 days were observed (Favata et al. 2005; Wolk et al. 2005). Spectral analysis of the flaring sources shows peak temperatures from $40 \mathrm{MK}$ up to $100 \mathrm{MK}$ for the strongest flares, and peak luminosities between $4 \times 10^{30}$ and $5 \times 10^{31} \mathrm{erg} \mathrm{s}^{-1}$. On the other hand, the flares observed on the two TMC non-members show significantly shorter rise and decay times ( $\lesssim 1 \mathrm{ks}$ and $\sim 2 \mathrm{ks}$, respectively), and peak temperature in the range $20-40 \mathrm{MK}$.

For four of the flaring sources (the WTTS HBC 427 and V410 Tau, the CTTS BP Tau, and the non-member 2MASS J04195676+2714488), we could perform a detailed analysis of the decay phase using the method by Reale et al. (1997); the method was also applied to V819 Tau, for which, however, only two points in the decay are available. In all these cases we find that significant residual heating must be present during the decay, governing the observed light curve evolution. For the other flaring sources, we do not have enough intervals in the decay phase to apply the method, and we could only estimate upper limits to the loop size using the formula for a freely decaying loop. The loop size is fully constrained only for the CTTS BP Tau, which has $L=1.2 \times 10^{11} \mathrm{~cm}$, comparable to the stellar radius, and for the WTTS HBC 427, which has $L=2.4 \times 10^{11} \mathrm{~cm}$ corresponding to $\sim 2 R_{\star}$; the latter star has the longest decay time, and hence the longest loop size, among the sources for which the entire flare evolution is observed. The other TMC members have loop lengths in the range $4-7 \times 10^{10} \mathrm{~cm}$, smaller than or comparable to the stellar radius. For the sources with continuous decay, only one (HQ Tau) has a steep slope, compatible with a freely-decaying loop with no additional heating after the initial ignition; for this star we find $L \sim 7 \times 10^{11} \mathrm{~cm}$. The other four sources have upper limits between $1-5 \times 10^{11} \mathrm{~cm}$, comparable to the stellar radius for the two WTTS, but equal to $\sim 4 R_{\star}$ for the two CTTS (FS Tau and $\mathrm{DH}$ Tau). Finally, for the TMC non-members we find loop sizes in the range $1 \times 10^{9}-3 \times 10^{10} \mathrm{~cm}$.

We mention that flare characteristics similar to those found here for TMC members are also observed for the CTTS
SU Aur (XEST-26-067, Franciosini et al. 2007), which showed three flares during the observation with rise and decay times of $\sim 6 \mathrm{ks}$ and $\sim 5-9 \mathrm{ks}$, respectively, and peak temperatures of $\sim 50-140$ MK. The data do not allow a detailed flare analysis, however, using the parameters reported by Franciosini et al. (2007) we derive upper limits to the loop semi-length of $\sim 1.1-1.8 \times 10^{11} \mathrm{~cm}$, comparable to the stellar radius.

Previous observations of PMS stars have shown the presence of compact flaring structures, with $L \lesssim R_{\star}$, similar to what is observed in active late-type stars (Favata et al. 2001; Grosso et al. 2004; Giardino et al. 2006; Güdel et al. 2004; Reale et al. 2004). Favata et al. (2005) analyzed a sample of strong flares detected in the COUP survey, finding both compact structures, of size shorter than a stellar radius, and very extended structures, with lengths of 5-20 $R_{\star}$. Such long structures possibly represent magnetic loops connecting the stellar surface with the circumstellar disk. Our sample generally shows loops of size comparable to or smaller than the stellar radius: the longest loop with a fully constrained size is about 2 stellar radii in length, which is compatible with a loop anchored on the stellar surface. We stress that this loop has been observed on a WTTS, which should not possess a circumstellar disk, and that a similar loop size $\left(\sim 1.6 R_{\star}\right)$ has been found also on the WTTS V827 Tau by Giardino et al. (2006). A possible hint for large loops of size $\sim 4 R_{\star}$ is found for the two CTTS with long-lasting decay, suggesting that these stars might indeed have loops connecting the star and the disk. However, the estimated size is highly uncertain, since we are observing only a small part of the decay; therefore we cannot draw any definitive conclusion on the size of the emitting structures.

Finally, we have studied two sources showing possible rotational modulation with amplitudes of $\sim 15 \%$, and two other sources (one unrelated to the TMC) with flare-like variability superimposed on a slow modulation or on a flare decay. Except for the last case, we do not find significant variations in the plasma temperatures, and the observed variability is mainly determined by variations of the emission measures.

Acknowledgements. E.F., I.P., and G.M. would like to thank F. Reale for useful discussions on the interpretation of flare variability. We acknowledge financial support by the International Space Science Institute (ISSI) in Bern, Switzerland to the XMM-Newton XEST team. The Palermo group acknowledges financial contribution from contract ASI-INAF I/023/05/0. X-ray astronomy research at PSI has been supported by the Swiss National Science Foundation (grants 20-66875.01 and 20-109255/1). M.A. acknowledges support from NASA grant NNG05GF92G. In addition, he acknowledges support from a Swiss National Science Foundation Professorship (PP002-110504). This research is based on observations obtained with XMM-Newton, an ESA science mission with instruments and contributions directly funded by ESA Member States and NASA.

\section{References}

Anders, E., \& Grevesse, N. 1989, Geochim. Cosmochim. Acta, 53, 197 Argiroffi, C., Drake, J. J., Maggio, A., et al. 2004, ApJ, 609, 925 Audard, M., Güdel, M., \& Mewe, R. 2001, A\&A, 365, L318

Audard, M., Güdel, M., Skinner, S. L., et al. 2005, ApJ, 635, L81 Favata, F., \& Micela, G. 2003, Sp. Sci. Rev., 108, 577

Favata, F., Micela, G., \& Reale, F. 2001, A\&A, 375, 485

Favata, F., Giardino, G., Micela, G., Sciortino, S., \& Damiani, F. 2003, A\&A, 403, 187

Favata, F., Flaccomio, E., Reale, F., et al. 2005, ApJS, 160, 469

Feigelson, E. D., \& Montmerle, T. 1999, ARA\&A, 37, 363

Flaccomio, E., Micela, G., \& Sciortino, S. 2003, A\&A, 397, 611

Flaccomio, E., Micela, G., Sciortino, S., et al. 2005, ApJS, 160, 450

Franciosini, E., Pallavicini, R., \& Sanz-Forcada, J. 2006, A\&A, 446, 501

Franciosini, E., Scelsi, L., Pallavicini, R., \& Audard, M. 2007, A\&A, submitted

Giardino, G., Favata, F., Micela, M., \& Reale, F. 2004, A\&A, 413, 669

Giardino, G., Favata, F., Silva, B., et al. 2006, A\&A, 453, 241 
Grosso, N., Montmerle, T., Feigelson, E. D., \& Forbes, T. G. 2004, A\&A, 419, 653

Güdel, M. 2004, A\&AR, 12, 71

Güdel, M., Audard, A., Reale, F., Skinner, S. L., \& Linsky, J. L. 2004, A\&A, 416, 713

Güdel, M., Briggs, K. R., Arzner, K., et al. 2007a, A\&A, 468, 353

Güdel, M., Skinner, S. L., Mel'nikov, S. Y., et al. 2007b, A\&A, 468, 529

Hamaguchi, K., Yamauchi, S., \& Koyama, K. 2005, ApJ, 618, 360

Herbig, G. H., Vrba, F. J., \& Rydgren, A. E. 1986, AJ, 91, 575

Kastner, J. H., Huenemorder, D. P., Schulz, N. S., Canizares, C. R., \& Weintraub, D. A. 2002, ApJ, 567, 434

Kastner, J. H., Richmond, M., Grosso, N., et al. 2004, Nature, 430, 429

Kastner, J. H., Richmond, M., Grosso, N., et al. 2006, ApJ, 648, L43

Kopp, R. A., \& Poletto, G. 1984, Sol. Phys., 93, 351

Leinert, C., Richichi, A., \& Haas, M. 1997, A\&A, 318, 472

Pneuman, G. W. 1982, Sol. Phys., 78, 229

Preibisch, T., Kim, Y.-C., Favata, F., et al. 2005, ApJS, 160, 401

Reale, F., Betta, R., Peres, G., Serio, S., \& McTiernan, J. 1997, A\&A, 325, 782

Reale, F., Güdel, M., Peres, G., \& Audard, M. 2004, A\&A, 416, 733

Scelsi, L., Maggio, A., Peres, G., \& Pallavicini, R. 2005, A\&A, 432, 671
Scelsi, L., Maggio, A., Micela, G., et al. 2007, A\&A, 468, 405

Schmitt, J. H. M. M., Robrade, J., Ness, J.-U., Favata, F., \& Stelzer, B. 2005, A\&A, 432, L35

Serio, S., Reale, F., Jakimiec, J., Sylwester, B., \& Sylwester, J. 1991, A\&A, 241, 197

Siess, L., Dufour, E., \& Forestini, M. 2000, A\&A, 358, 593

Smith, K. W., Balega, Y. Y., Duschl, W. J., et al. 2005, A\&A, 431, 307

Stelzer, B., Flaccomio, F., Briggs, K., et al. 2007, A\&A, 468, 463

Stelzer, B., Micela, G., Hamaguchi, K., \& Schmitt, J. H. M. M. 2006, A\&A, 457, 223

Stelzer, B., \& Neuhäuser, R. 2001, A\&A, 377, 538

Stelzer, B., \& Schmitt, J. H. M. M. 2004, A\&A, 418, 687

Swartz, D. A., Drake, J. J., Elsner, R. F., et al. 2005, ApJ, 628, 811

Telleschi, A., Güdel, M., Briggs, K., et al. 2005, ApJ, 622, 653

Telleschi, A., Güdel, M., Briggs, K. R., et al. 2007a, A\&A, 428, 541

Telleschi, A., Güdel, M., Briggs, K. R., et al. 2007b, A\&A, 428, 425

Walter, F. M., Brown, A., Mathieu, R. D., Myers, P. C., \& Vrba, F. J. 1988, AJ, 96, 297

Wolk, S. J., Harnden, F. R., J., Flaccomio, E., et al. 2005, ApJS, 160, 423 
E. Franciosini et al.: X-ray bright variable sources in the Taurus molecular cloud, Online Material $p 1$

\section{Online Material}


E. Franciosini et al.: X-ray bright variable sources in the Taurus molecular cloud, Online Material p 2

Table 2. Best-fit values of the spectral model parameters. Errors are $90 \%$ confidence ranges for one interesting parameter; where errors are not given, the parameter was held fixed to the tabulated value.

\begin{tabular}{|c|c|c|c|c|c|c|c|c|c|c|c|}
\hline XEST-ID & Optical ID & $\begin{array}{r}\text { Block } \\
\#\end{array}$ & $\begin{array}{c}N_{\mathrm{H}} \\
10^{21} \mathrm{~cm}^{-2}\end{array}$ & $\begin{array}{c}T_{1} \\
\mathrm{keV}\end{array}$ & $\begin{array}{c}T_{2} \\
\mathrm{keV}\end{array}$ & $\begin{array}{l}\mathrm{EM}_{1} \\
\quad 10^{53}\end{array}$ & $\begin{array}{r}\mathrm{EM}_{2} \\
\mathrm{~cm}^{-3}\end{array}$ & $\chi_{\mathrm{r}}^{2}$ & d.o.f. & $\begin{array}{c}F_{\mathrm{X}}^{a} / 10^{-12} \\
\operatorname{erg~cm}^{-2} \mathrm{~s}^{-1}\end{array}$ & $\begin{array}{l}\log L_{X}^{a} \\
\operatorname{erg~s}^{-1}\end{array}$ \\
\hline 04-016 & V830 Tau & $\begin{array}{l}1 \\
2 \\
3 \\
4 \\
5 \\
6\end{array}$ & $\begin{array}{c}0.33_{-0.11}^{+0.10} \\
0.34_{-0.24}^{+0.25} \\
0.46_{-0.16}^{+0.26} \\
0.33_{-0.12}^{+0.12} \\
0.32_{-0.13}^{+0.14} \\
0.24_{-0.18}^{+0.20}\end{array}$ & $\begin{array}{l}0.79_{-0.07}^{+0.06} \\
0.51_{-0.11}^{+0.24} \\
0.98_{-0.21}^{+0.08} \\
0.67_{-0.06}^{+0.07} \\
0.76_{-0.09}^{+0.06} \\
0.65_{-0.27}^{+0.11}\end{array}$ & $\begin{array}{l}2.08_{-0.25}^{+0.44} \\
1.88_{-0.25}^{+0.26} \\
2.80_{-1.19}^{+2.66} \\
1.86_{-0.20}^{+0.25} \\
1.95_{-0.41}^{+0.49} \\
1.93_{-0.30}^{+0.52}\end{array}$ & $\begin{array}{l}2.30_{-0.49}^{+0.45} \\
1.29_{-0.42}^{+0.54} \\
2.85_{-1.51}^{+0.82} \\
1.41_{-0.24}^{+0.21} \\
1.25_{-0.26}^{+0.28} \\
0.96_{-0.21}^{+0.24}\end{array}$ & $\begin{array}{c}5.64_{-0.66}^{+0.66} \\
5.08_{-0.73}^{+0.54} \\
2.63_{-0.92}^{+2.38} \\
2.87_{-0.35}^{+0.28} \\
2.07_{-0.35}^{+0.19} \\
1.81_{-0.35}^{+0.28}\end{array}$ & $\begin{array}{l}1.06 \\
1.21 \\
1.09 \\
1.12 \\
1.16 \\
1.10\end{array}$ & $\begin{array}{r}289 \\
99 \\
96 \\
193 \\
143 \\
94\end{array}$ & $\begin{array}{l}3.59 \\
2.80 \\
2.56 \\
1.87 \\
1.47 \\
1.21\end{array}$ & $\begin{array}{l}30.93 \\
30.82 \\
30.78 \\
30.64 \\
30.54 \\
30.45\end{array}$ \\
\hline 09-026 & HQ Tau & $\begin{array}{l}1 \\
2 \\
3 \\
4\end{array}$ & $\begin{array}{r}4.94_{-1.12}^{+1.36} \\
4.52_{-0.43}^{+0.49} \\
3.58_{-0.00}^{+1.03} \\
4.68_{-0.59}^{+0.74} \\
\end{array}$ & $\begin{array}{c}0.61_{-0.24}^{+0.16} \\
0.76_{-0.06}^{+0.07} \\
0.71_{-0.15}^{+0.12} \\
0.74_{-0.14}^{+0.10}\end{array}$ & $\begin{array}{r}2.90_{-0.62}^{+1.11} \\
2.00_{-0.36}^{+0.33} \\
1.92_{-0.35}^{+0.56} \\
1.62_{-0.25}^{+0.71} \\
\end{array}$ & $\begin{array}{l}4.89_{-1.98}^{+3.90} \\
3.17_{-0.66}^{+0.85} \\
1.98_{-0.66}^{+1.27} \\
2.12_{-0.68}^{+0.73}\end{array}$ & $\begin{array}{r}3.48_{-0.75}^{+1.22} \\
3.25_{-0.54}^{+0.49} \\
2.52_{-0.59}^{+0.66} \\
2.42_{-0.85}^{+0.73} \\
\end{array}$ & $\begin{array}{l}0.80 \\
1.05 \\
0.98 \\
1.08\end{array}$ & $\begin{array}{r}92 \\
238 \\
93 \\
83\end{array}$ & $\begin{array}{l}3.88 \\
2.84 \\
1.97 \\
1.94\end{array}$ & $\begin{array}{l}30.96 \\
30.82 \\
30.67 \\
30.66\end{array}$ \\
\hline $11-057$ & FS Tau & $\begin{array}{l}1 \\
2 \\
3\end{array}$ & $\begin{array}{l}15.99_{-1.90}^{+1.96} \\
13.14_{-1.76}^{+1.87} \\
11.18_{-1.32}^{+1.45}\end{array}$ & $\begin{array}{l}\cdots \\
\cdots\end{array}$ & $\begin{array}{l}3.65_{-0.55}^{+0.78} \\
3.61_{-0.60}^{+0.90} \\
3.08_{-0.48}^{+0.58}\end{array}$ & $\begin{array}{l}\cdots \\
\cdots\end{array}$ & $\begin{array}{c}4.73_{-0.68}^{+0.73} \\
2.87_{-0.42}^{+0.52} \\
1.08_{-0.14}^{+0.19}\end{array}$ & $\begin{array}{l}0.91 \\
0.96 \\
0.93\end{array}$ & $\begin{array}{r}108 \\
82 \\
151\end{array}$ & $\begin{array}{l}2.68 \\
1.61 \\
0.59\end{array}$ & $\begin{array}{l}30.80 \\
30.58 \\
30.14\end{array}$ \\
\hline $12-040$ & DN Tau & $\begin{array}{l}1 \\
2 \\
3\end{array}$ & $\begin{array}{c}0.81_{-0.29}^{+0.31} \\
0.66_{-0.18}^{+0.14} \\
0.65_{-0.15}^{+0.15}\end{array}$ & $\begin{array}{c}0.76_{-0.10}^{+0.08} \\
0.66_{-0.10}^{+0.10} \\
0.79_{-0.05}^{+0.05}\end{array}$ & $\begin{array}{l}2.56_{-0.79}^{+5.11} \\
1.67_{-0.14}^{+0.49} \\
2.32_{-0.45}^{+0.59}\end{array}$ & $\begin{array}{c}0.66_{-0.16}^{+0.19} \\
0.42_{-0.07}^{+0.07} \\
0.54_{-0.09}^{+0.07}\end{array}$ & $\begin{array}{c}0.66_{-0.21}^{+0.21} \\
0.56_{-0.12}^{+0.07} \\
0.56_{-0.09}^{+0.12}\end{array}$ & $\begin{array}{l}0.95 \\
1.15 \\
0.93\end{array}$ & $\begin{array}{r}65 \\
201 \\
169\end{array}$ & $\begin{array}{l}0.61 \\
0.42 \\
0.50\end{array}$ & $\begin{array}{l}30.16 \\
29.99 \\
30.07\end{array}$ \\
\hline $15-040$ & DH Tau & $\begin{array}{l}1 \\
2 \\
3 \\
4 \\
5\end{array}$ & $\begin{array}{l}1.89_{-0.14}^{+0.14} \\
1.86_{-0.16}^{+0.17} \\
1.87_{-0.15}^{+0.16} \\
1.69_{-0.16}^{+0.18} \\
2.03_{-0.53}^{+1.16}\end{array}$ & $\begin{array}{l}0.74_{-0.05}^{+0.05} \\
0.80_{-0.05}^{+0.05} \\
0.75_{-0.05}^{+0.04} \\
0.76_{-0.05}^{+0.04} \\
0.61_{-0.22}^{+0.11}\end{array}$ & $\begin{array}{l}2.06_{-0.14}^{+0.16} \\
2.05_{-0.20}^{+0.27} \\
1.95_{-0.16}^{+0.20} \\
2.06_{-0.31}^{+0.34} \\
1.69_{-0.33}^{+0.61}\end{array}$ & $\begin{array}{l}3.27_{-0.42}^{+0.47} \\
3.25_{-0.45}^{+0.47} \\
2.61_{-0.33}^{+0.35} \\
2.42_{-0.31}^{+0.33} \\
2.56_{-0.80}^{+2.28}\end{array}$ & $\begin{array}{l}6.04_{-0.52}^{+0.52} \\
4.30_{-0.52}^{+0.52} \\
3.57_{-0.35}^{+0.35} \\
2.35_{-0.33}^{+0.35} \\
2.09_{-0.56}^{+0.75}\end{array}$ & $\begin{array}{l}0.96 \\
1.07 \\
0.90 \\
0.98 \\
1.04\end{array}$ & $\begin{array}{r}412 \\
298 \\
357 \\
270 \\
57\end{array}$ & $\begin{array}{l}4.18 \\
3.36 \\
2.74 \\
2.11 \\
1.98\end{array}$ & $\begin{array}{l}30.99 \\
30.90 \\
30.81 \\
30.70 \\
30.67\end{array}$ \\
\hline $17-066$ & JH 108 & $\begin{array}{l}1 \\
2 \\
3\end{array}$ & $\begin{array}{l}2.07_{-0.35}^{+0.40} \\
2.12_{-0.29}^{+0.38} \\
2.09_{-0.38}^{+0.46}\end{array}$ & $\begin{array}{l}1.29_{-0.37}^{+0.72} \\
0.86_{-0.15}^{+0.17} \\
0.73_{-0.08}^{+0.08}\end{array}$ & $\begin{array}{c}3.94_{-3.60}^{+10.04} \\
2.14_{-0.31}^{+0.57} \\
2.43_{-0.62}^{+1.36}\end{array}$ & $\begin{array}{l}0.89_{-0.79}^{+1.79} \\
0.78_{-0.43}^{+0.45} \\
0.59_{-0.14}^{+0.12}\end{array}$ & $\begin{array}{l}1.81_{-1.34}^{+0.73} \\
2.63_{-0.54}^{+0.52} \\
0.89_{-0.26}^{+0.09}\end{array}$ & $\begin{array}{l}0.84 \\
1.12 \\
1.23\end{array}$ & $\begin{array}{r}93 \\
120 \\
97\end{array}$ & $\begin{array}{l}1.42 \\
1.57 \\
0.64\end{array}$ & $\begin{array}{l}30.52 \\
30.57 \\
30.18\end{array}$ \\
\hline 21-039 & HD 283572 & $\begin{array}{l}1 \\
2 \\
3 \\
4 \\
5 \\
6 \\
7\end{array}$ & $\begin{array}{l}0.66_{-0.10}^{+0.11} \\
0.72_{-0.07}^{+0.05} \\
0.59_{-0.02}^{+0.04} \\
0.64_{-0.19}^{+0.18} \\
0.68_{-0.05}^{+0.06} \\
0.64_{-0.06}^{+0.06} \\
0.66_{-0.07}^{+0.07}\end{array}$ & $\begin{array}{l}0.75_{-0.03}^{+0.03} \\
0.74_{-0.02}^{+0.01} \\
0.76_{-0.01}^{+0.01} \\
0.70_{-0.06}^{+0.05} \\
0.75_{-0.01}^{+0.02} \\
0.73_{-0.03}^{+0.02} \\
0.74_{-0.02}^{+0.02}\end{array}$ & $\begin{array}{l}1.94_{-0.20}^{+0.22} \\
2.03_{-0.10}^{+0.07} \\
2.22_{-0.12}^{+0.08} \\
1.83_{-0.16}^{+0.19} \\
1.87_{-0.08}^{+0.07} \\
1.67_{-0.08}^{+0.06} \\
1.86_{-0.08}^{+0.09}\end{array}$ & $\begin{array}{l}5.48_{-1.06}^{+1.29} \\
4.61_{-0.66}^{+0.40} \\
3.34_{-0.45}^{+0.33} \\
3.86_{-1.15}^{+1.25} \\
4.14_{-0.38}^{+0.56} \\
3.48_{-0.42}^{+0.47} \\
4.12_{-0.66}^{+0.59}\end{array}$ & $\begin{array}{l}6.37_{-0.68}^{+0.63} \\
8.23_{-0.24}^{+0.38} \\
8.96_{-0.19}^{+0.35} \\
7.50_{-0.66}^{+0.66} \\
6.23_{-0.28}^{+0.26} \\
6.11_{-0.24}^{+0.40} \\
5.97_{-0.31}^{+0.38}\end{array}$ & $\begin{array}{l}0.99 \\
1.05 \\
1.09 \\
0.99 \\
1.05 \\
0.98 \\
1.16\end{array}$ & $\begin{array}{l}347 \\
825 \\
782 \\
220 \\
715 \\
616 \\
553\end{array}$ & $\begin{array}{l}5.46 \\
6.05 \\
6.21 \\
5.39 \\
4.91 \\
4.50 \\
4.74\end{array}$ & $\begin{array}{l}31.11 \\
31.15 \\
31.16 \\
31.10 \\
31.06 \\
31.02 \\
31.05\end{array}$ \\
\hline $22-047$ & XZ Tau & $\begin{array}{l}1 \\
2 \\
3 \\
4 \\
5\end{array}$ & $\begin{array}{l}2.45_{-0.44}^{+0.61} \\
2.05_{-0.39}^{+0.47} \\
1.91_{-0.33}^{+0.39} \\
2.22_{-0.21}^{+0.23} \\
2.56_{-0.26}^{+0.29}\end{array}$ & $\begin{array}{l}0.73_{-0.11}^{+0.08} \\
0.82_{-0.09}^{+0.15} \\
0.77_{-0.12}^{+0.13} \\
0.78_{-0.09}^{+0.13} \\
0.79_{-0.09}^{+0.09}\end{array}$ & $\begin{array}{l}3.44_{-0.95}^{+2.31} \\
5.72_{-1.71}^{+4.49} \\
6.03_{-1.06}^{+2.06} \\
4.42_{-0.45}^{+0.64} \\
3.50_{-0.42}^{+0.52}\end{array}$ & $\begin{array}{l}0.45_{-0.09}^{+0.12} \\
0.49_{-0.12}^{+0.16} \\
0.45_{-0.14}^{+0.14} \\
0.49_{-0.12}^{+0.12} \\
0.82_{-0.19}^{+0.19}\end{array}$ & $\begin{array}{l}0.31_{-0.07}^{+0.09} \\
0.68_{-0.09}^{+0.12} \\
1.25_{-0.09}^{+0.16} \\
1.83_{-0.12}^{+0.12} \\
2.23_{-0.16}^{+0.21}\end{array}$ & $\begin{array}{l}1.44 \\
0.90 \\
1.04 \\
0.95 \\
0.94\end{array}$ & $\begin{array}{r}75 \\
80 \\
143 \\
278 \\
230\end{array}$ & $\begin{array}{l}0.36 \\
0.65 \\
1.00 \\
1.30 \\
1.60\end{array}$ & $\begin{array}{l}29.93 \\
30.18 \\
30.37 \\
30.49 \\
30.58\end{array}$ \\
\hline 22-089 & L1551 51 & $\begin{array}{l}1 \\
2 \\
3\end{array}$ & $\begin{array}{c}0.87_{-0.24}^{+0.28} \\
1.00_{-0.21}^{+0.69} \\
0.62_{-0.18}^{+0.21}\end{array}$ & $\begin{array}{c}0.65_{-0.17}^{+0.14} \\
0.62_{-0.15}^{+0.06} \\
0.63_{-0.06}^{+0.05}\end{array}$ & $\begin{array}{l}1.50_{-0.23}^{+0.49} \\
1.25_{-0.21}^{+0.59} \\
1.37_{-0.23}^{+0.63}\end{array}$ & $\begin{array}{l}1.01_{-0.26}^{+0.31} \\
1.13_{-0.26}^{+0.31} \\
0.85_{-0.16}^{+0.12}\end{array}$ & $\begin{array}{c}1.53_{-0.47}^{+0.24} \\
0.61_{-0.24}^{+0.21} \\
0.38_{-0.12}^{+0.14}\end{array}$ & $\begin{array}{l}0.92 \\
1.19 \\
0.95\end{array}$ & $\begin{array}{r}63 \\
90 \\
101\end{array}$ & $\begin{array}{l}1.08 \\
0.72 \\
0.59\end{array}$ & $\begin{array}{l}30.40 \\
30.23 \\
30.14\end{array}$ \\
\hline
\end{tabular}


E. Franciosini et al.: X-ray bright variable sources in the Taurus molecular cloud, Online Material p 3

Table 2. continued.

\begin{tabular}{|c|c|c|c|c|c|c|c|c|c|c|c|}
\hline XEST-ID & Optical ID & $\begin{array}{r}\text { Block } \\
\#\end{array}$ & $\begin{array}{c}N_{\mathrm{H}} \\
10^{21} \mathrm{~cm}^{-2}\end{array}$ & $\begin{array}{c}T_{1} \\
\mathrm{keV}\end{array}$ & $\begin{array}{c}T_{2} \\
\mathrm{keV}\end{array}$ & \multicolumn{2}{|c|}{$10^{53} \mathrm{~cm}^{-3}$} & $\chi_{\mathrm{r}}^{2}$ & d.o.f. & $\begin{array}{c}F_{\mathrm{X}}^{a} / 10^{-12} \\
\mathrm{erg} \mathrm{cm}^{-2} \mathrm{~s}^{-1}\end{array}$ & $\begin{array}{l}\log L_{X}^{a} \\
\operatorname{erg~s}^{-1}\end{array}$ \\
\hline \multirow[t]{17}{*}{$23-032+24-028$} & V410 Tau & 1 & 0.10 & $0.74_{-0.02}^{+0.02}$ & $1.70_{-0.08}^{+0.14}$ & $1.79_{-0.14}^{+0.12}$ & $2.00_{-0.14}^{+0.14}$ & 1.27 & 685 & 1.63 & 30.58 \\
\hline & & 2 & $0.11_{-0.05}^{+0.05}$ & $0.75_{-0.03}^{+0.02}$ & $2.05_{-0.15}^{+0.25}$ & $1.90_{-0.16}^{+0.14}$ & $2.80_{-0.26}^{+0.14}$ & 1.07 & 540 & 2.10 & 30.69 \\
\hline & & 3 & $0.09_{-0.06}^{+0.05}$ & $0.75_{-0.03}^{+0.03}$ & $2.30_{-0.26}^{+0.22}$ & $1.90_{-0.12}^{+0.16}$ & $3.39_{-0.24}^{+0.31}$ & 1.18 & 498 & 2.43 & 30.76 \\
\hline & & 4 & $0.15_{-0.05}^{+0.05}$ & $0.79_{-0.02}^{+0.03}$ & $2.48_{-0.20}^{+0.23}$ & $2.52_{-0.19}^{+0.19}$ & $3.60_{-0.26}^{+0.26}$ & 1.06 & 494 & 2.84 & 30.82 \\
\hline & & 5 & $0.19_{-0.07}^{+0.07}$ & $0.77_{-0.03}^{+0.02}$ & $2.33_{-0.26}^{+0.20}$ & $3.01_{-0.28}^{+0.31}$ & $4.04_{-0.38}^{+0.42}$ & 1.08 & 373 & 3.25 & 30.88 \\
\hline & & 6 & $0.25_{-0.04}^{+0.07}$ & $0.75_{-0.02}^{-0.03}$ & $2.10_{-0.12}^{+0.26}$ & $3.53_{-0.24}^{+0.31}$ & $4.56_{-0.42}^{+0.38}$ & 1.02 & 571 & 3.63 & 30.93 \\
\hline & & 7 & $0.29_{-0.14}^{-0.04}$ & $0.82_{-0.05}^{-0.02}$ & $3.67_{-0.92}^{+0.12}$ & $4.47_{-0.66}^{-0.63}$ & $4.42_{-0.78}^{-0.42}$ & 1.00 & 144 & 4.41 & 31.02 \\
\hline & & 8 & $0.33_{-0.08}^{-0.09}$ & $0.77_{-0.04}^{-0.05}$ & $2.50_{-0.25}^{+0.92}$ & $3.83_{-0.42}^{+0.46}$ & $\begin{array}{l}6.70_{-0.59}^{+0.59} \\
69\end{array}$ & 0.87 & 355 & 4.95 & 31.07 \\
\hline & & 9 & $0.35_{-0.07}^{+0.08}$ & $0.78_{-0.04}^{+0.04}$ & $2.02_{-0.16}^{+0.25}$ & $3.25_{-0.38}^{+0.40}$ & $5.67_{-0.56}^{+0.52}$ & 1.04 & 369 & 3.99 & 30.97 \\
\hline & & 10 & $0.37_{-0.08}^{+0.09}$ & $0.77_{-0.04}^{-0.04}$ & $2.07_{-0.21}^{+0.46}$ & $3.27_{-0.40}^{+0.48}$ & $4.35_{-0.63}^{+0.46}$ & 0.88 & 292 & 3.40 & 30.90 \\
\hline & & 11 & $0.21_{-0.06}^{+0.08}$ & $0.76_{-0.04}^{+0.04}$ & $1.90_{-0.17}^{+0.21}$ & $3.03_{-0.28}^{+0.20}$ & $3.22_{-0.35}^{+0.03}$ & 1.15 & 377 & 4.27 & 31.00 \\
\hline & & 12 & $\begin{array}{l}0.20_{-0.07}^{+0.06} \\
0.09\end{array}$ & $0.77_{-0.04}^{+0.04}$ & $1.99_{-0.23}^{+0.34}$ & $2.66_{-0.31}^{+0.38}$ & $2.73_{-0.40}^{+0.38}$ & 1.00 & 291 & 2.38 & 30.75 \\
\hline & & 13 & $0.14_{-0.03}^{+0.03}$ & $0.73_{-0.01}^{+0.02}$ & $1.79_{-0.10}^{+0.11}$ & $1.98_{-0.09}^{+0.12}$ & $2.52_{-0.14}^{+0.07}$ & 1.15 & 818 & 1.94 & 30.66 \\
\hline & & 14 & $0.07_{-0.06}^{+0.06}$ & $0.77_{-0.06}^{+0.03}$ & $1.75_{-0.20}^{+0.110}$ & $1.55_{-0.28}^{+0.16}$ & $2.09_{-0.19}^{+0.35}$ & 1.03 & 448 & 1.58 & 30.57 \\
\hline & & 15 & $0.12_{-0.10}^{+0.10}$ & $\begin{array}{l}0.79_{-0.05}^{+0.00} \\
\end{array}$ & $2.49_{-0.37}^{+0.54}$ & $1.74_{-0.24}^{-0.28}$ & $2.66_{-0.35}^{+0.35}$ & 1.03 & 212 & 2.06 & 30.69 \\
\hline & & 16 & $0.10^{-0.10}$ & $0.58_{-0.17}^{-0.05}$ & $1.53_{-0.20}^{+0.18}$ & $0.99_{-0.28}^{+0.28}$ & $2.78_{-0.35}^{+0.35}$ & 1.19 & 93 & 1.58 & 30.57 \\
\hline & & 17 & 0.10 & $0.76_{-0.04}^{+0.04}$ & $1.81_{-0.16}^{+0.32}$ & $1.41_{-0.16}^{+0.19}$ & $1.81_{-0.24}^{+0.21}$ & 0.91 & 311 & 1.41 & 30.52 \\
\hline \multirow[t]{12}{*}{$23-047+24-040$} & V892 Tau & 1 & $8.96_{-1.70}^{+1.67}$ & $0.82_{-0.13}^{+0.22}$ & $2.98_{-0.47}^{+0.84}$ & $3.15_{-1.79}^{+2.78}$ & $3.39_{-0.73}^{+0.63}$ & 1.10 & 254 & 3.13 & 30.87 \\
\hline & & 2 & $10.51_{-372}^{+1.44}$ & $0.69_{-017}^{+0.38}$ & $2.93_{-062}^{+1.713}$ & $5.86_{-294}^{+7.31}$ & $4.99_{-1.67}^{+1.13}$ & 0.79 & 77 & 5.08 & 31.08 \\
\hline & & 3 & $8.90_{-0.77}^{+1.10}$ & $0.92_{-0.16}^{+0.11}$ & $2.87_{-0.36}^{-0.52}$ & $3.25_{-1.08}^{+1.94}$ & $4.00_{-0.73}^{+0.67}$ & 0.97 & 338 & 3.46 & 30.91 \\
\hline & & 4 & $8.14_{-0.92}^{+1.13}$ & $1.06_{-0.18}^{+0.16}$ & $3.02_{-0.41}^{+0.76}$ & $2.89_{-1.39}^{+1.08}$ & $4.99_{-1.18}^{+0.03}$ & 1.05 & 194 & 3.86 & 30.96 \\
\hline & & 5 & $9.59_{-1.06}^{+1.22}$ & $0.81_{-0.12}^{-0.18}$ & $2.37_{-0.27}^{+0.41}$ & $4.59_{-1.83}^{+2.39}$ & $4.19_{-0.80}^{+0.18}$ & 1.13 & 242 & 3.99 & 30.97 \\
\hline & & 6 & $12.33_{-1.59}^{+1.60}$ & $0.38_{-0.05}^{+0.07}$ & $1.84_{-0.16}^{+0.18}$ & $13.90_{-6.87}^{+12.80}$ & $5.13_{-0.59}^{+0.80}$ & 0.97 & 232 & 7.52 & 31.25 \\
\hline & & 7 & $11.05_{-1.85}^{+1.24}$ & $0.49_{-0.08}^{+0.19}$ & $1.95_{-0.20}^{+0.40}$ & $6.00_{-3.41}^{+3.53}$ & $3.69_{-0.92}^{+0.61}$ & 1.05 & 295 & 4.09 & 30.98 \\
\hline & & 8 & $10.73_{-0.67}^{+0.85}$ & $0.49^{-0.08}$ & $5.71_{-1.32}^{+0.20}$ & $6.00^{-3.41}$ & $6.21_{-0.61}^{-0.92}$ & 1.03 & 90 & 6.38 & 31.18 \\
\hline & & 9 & $\begin{array}{r}-0.67 \\
12.07_{-1.05}^{+1.20}\end{array}$ & 0.49 & $8.04_{-2.21}^{+4.32}$ & 6.00 & $\begin{array}{r}-0.61 \\
13.71_{-115}^{+1.58}\end{array}$ & 1.02 & 85 & 11.60 & 31.44 \\
\hline & & 10 & $11.68_{-0.86}^{-1.09}$ & 0.49 & $8.62_{-1.94}^{+4.12}$ & 6.00 & $18.04_{-1.15}^{-1.45}$ & 0.72 & 124 & 14.59 & 31.54 \\
\hline & & 11 & $11.53_{-0.78}^{+0.79}$ & 0.49 & $8.23_{-1.59}^{+2.94}$ & 6.00 & $21.38_{-1.34}^{+1.48}$ & 0.99 & 181 & 16.72 & 31.59 \\
\hline & & 12 & $\begin{array}{l}11.26_{-0.26}^{+0.28} \\
0\end{array}$ & 0.49 & $7.24_{-0.59}^{+0.65}$ & 6.00 & $26.72_{-0.66}^{-0.73}$ & 0.99 & 862 & 20.08 & 31.67 \\
\hline \multirow[t]{4}{*}{$23-050+24-042$} & V410 X7 & 1 & $7.41_{-1.91}^{+5.79}$ & $0.77_{-0.53}^{+0.37}$ & $2.07_{-0.39}^{+2.38}$ & $0.33_{-0.24}^{+1.60}$ & $0.35_{-0.19}^{+0.09}$ & 1.16 & 130 & 0.30 & 29.85 \\
\hline & & 2 & $8.19_{-1.04}^{+1.91}$ & $1.36_{-0.036}^{+0.53}$ & $\begin{array}{r}-0.39 \\
5.43_{-147}^{+2.36}\end{array}$ & $\begin{array}{r}-0.24 \\
2.42^{+1.58}\end{array}$ & $\begin{array}{l}0.0 .19 \\
1.69^{+1.76}\end{array}$ & 0.92 & 105 & 2.07 & 30.69 \\
\hline & & 3 & $8.34_{-166}^{-1.44}$ & $0.68_{-0.04}^{+0.28}$ & $2.55_{-0.47}^{+0.49}$ & $0.63_{-0.52}^{+1.56}$ & $2.07_{-0.11}^{+0.08}$ & 0.97 & 139 & 1.29 & 30.48 \\
\hline & & 4 & $10.46_{-1.87}^{+4.81}$ & $0.68_{-0.44}^{+0.24}$ & $1.78_{-0.44}^{+1.44}$ & $2.02_{-1.32}^{+2.09}$ & $1.34_{-0.73}^{+1.69}$ & 1.06 & 116 & 1.45 & 30.53 \\
\hline \multirow[t]{7}{*}{$23-056+24-047$} & Hubble 4 & 1 & $2.32_{-0.09}^{+0.09}$ & $0.74_{-0.02}^{+0.02}$ & $1.81_{-0.15}^{+0.12}$ & $2.54_{-0.16}^{+0.16}$ & $2.49_{-0.19}^{+0.24}$ & 1.19 & 680 & 2.18 & 30.71 \\
\hline & & 2 & $1.58_{-0.32}^{+0.09}$ & $0.77_{-0.10}^{-0.02}$ & $\begin{array}{r}2.28_{-0.45}^{+0.15} \\
\end{array}$ & $1.53_{-0.35}^{+0.16}$ & $\begin{array}{r}-0.19 \\
1.76_{-0.38}^{+0.40}\end{array}$ & 0.96 & 89 & 1.50 & 30.55 \\
\hline & & 3 & $2.15_{-0.19}^{+0.32}$ & $0.76_{-0.05}^{+0.04}$ & $1.84_{-0.20}^{+0.25}$ & $2.07_{-0.26}^{-0.35}$ & $\begin{array}{r}2.07_{-0.28}^{+0.38} \\
\end{array}$ & 1.00 & 239 & 1.80 & 30.63 \\
\hline & & 4 & $2.24_{-0.13}^{+0.19}$ & $0.77_{-0.04}^{-0.03}$ & $2.08_{-0.15}^{+0.20}$ & $2.30_{-0.24}^{+0.26}$ & $2.80_{-0.31}^{+0.28}$ & 1.00 & 443 & 2.28 & 30.73 \\
\hline & & 5 & $2.09_{-0.16}^{+0.13}$ & $0.76_{-0.04}^{+0.04}$ & $1.87_{-0.21}^{+0.13}$ & $2.12_{-0.24}^{+0.24}$ & $2.14_{-0.28}^{+0.21}$ & 1.06 & 302 & 1.85 & 30.64 \\
\hline & & 6 & $2.15_{-0.13}^{+0.10}$ & $0.73_{-0.12}^{+0.04}$ & $1.63_{-0.13}^{+0.36}$ & $1.86_{-0.21}^{+0.34}$ & $1.88_{-0.33}^{+0.20}$ & 1.16 & 494 & 1.60 & 30.58 \\
\hline & & 7 & $2.29_{-0.28}^{+0.44}$ & $0.73_{-0.10}^{+0.05}$ & $2.63_{-0.58}^{+0.15}$ & $2.47_{-0.38}^{+0.41}$ & $1.79_{-0.38}^{+0.43}$ & 0.92 & 165 & 1.94 & 30.66 \\
\hline \multirow[t]{4}{*}{$23-074+24-061$} & V819 Tau & 1 & $2.11_{-0.20}^{+0.23}$ & $0.40_{-0.02}^{+0.02}$ & $1.31_{-0.07}^{+0.13}$ & $1.41_{-0.19}^{+0.26}$ & $0.92_{-0.07}^{+0.07}$ & 1.06 & 438 & 0.93 & 30.34 \\
\hline & & 2 & $2.24_{-0.51}^{+0.58}$ & $0.38_{-0.06}^{+0.02}$ & $1.66_{-0.19}^{+0.49}$ & $1.60_{-0.61}^{+0.85}$ & $2.38_{-0.035}^{+0.07}$ & 0.87 & 80 & 1.63 & 30.58 \\
\hline & & 3 & $\begin{array}{l}1.72_{-0.42}^{+0.49} \\
\end{array}$ & $\begin{array}{l}0.37_{-0.05}^{+0.06} \\
\end{array}$ & $1.22_{-0.11}^{+0.19}$ & $1.08_{-0.45}^{+0.61}$ & $1.18_{-0.16}^{+0.14}$ & 0.89 & 100 & 0.89 & 30.32 \\
\hline & & 4 & $2.23_{-0.34}^{+0.46}$ & $0.36_{-0.04}^{+0.03}$ & $1.20_{-0.12}^{+0.11}$ & $1.34_{-0.31}^{+0.45}$ & $0.89_{-0.12}^{+0.16}$ & 0.93 & 218 & 0.87 & 30.31 \\
\hline
\end{tabular}


E. Franciosini et al.: X-ray bright variable sources in the Taurus molecular cloud, Online Material p 4

Table 2. continued.

\begin{tabular}{|c|c|c|c|c|c|c|c|c|c|c|c|}
\hline XEST-ID & Optical ID & $\begin{array}{r}\text { Block } \\
\#\end{array}$ & $\begin{array}{c}N_{\mathrm{H}} \\
10^{21} \mathrm{~cm}^{-2}\end{array}$ & $\begin{array}{c}T_{1} \\
\mathrm{keV}\end{array}$ & $\begin{array}{c}T_{2} \\
\mathrm{keV}\end{array}$ & & $10^{53} \mathrm{~cm}^{-3}$ & $\chi_{\mathrm{r}}^{2}$ & d.o.f. & $\begin{array}{c}F_{\mathrm{X}}^{a} / 10^{-12} \\
\mathrm{erg} \mathrm{cm}^{-2} \mathrm{~s}^{-1}\end{array}$ & $\begin{array}{l}\log L_{\mathrm{X}}^{a} \\
\operatorname{erg~s}^{-1}\end{array}$ \\
\hline \multirow[t]{8}{*}{$26-072$} & HBC 427 & 1 & $0.20_{-0.19}^{+0.20}$ & $0.79_{-0.07}^{+0.06}$ & $2.14^{+0.90}$ & $1.51_{-0.28}^{+0.26}$ & $1.48^{+0.35}$ & 0.88 & 104 & 1.34 & 30.50 \\
\hline & & 2 & $0.29_{-028}^{+0.19}$ & $0.93_{-0.018}^{+0.07}$ & $6.18_{-2.05}^{+5.43}$ & $2.00_{-0.02}^{+0.98}$ & $5.39_{-0.82}^{+0.33}$ & 0.99 & 73 & 4.32 & 31.01 \\
\hline & & 3 & $\begin{array}{l}-0.28 \\
0.57_{-0.23}^{+0.28}\end{array}$ & $0.50_{-0.14}^{+0.18}$ & $\begin{array}{l}-2.05 \\
3.31_{-044}^{+0.75}\end{array}$ & $1.81_{-0.073}^{+0.82}$ & $10.89_{-1.01}^{-0.82}$ & 0.95 & 132 & 6.70 & 31.20 \\
\hline & & 4 & $0.49_{-0.26}^{+0.23}$ & $0.89_{-0.13}^{+0.14}$ & $2.88_{-0.64}^{+1.44}$ & $2.68_{-1.01}^{+1.73}$ & $6.68_{-1.62}^{+1.01}$ & 0.94 & 74 & 4.62 & 31.04 \\
\hline & & 5 & $0.44_{-0.13}^{-0.15}$ & $0.80_{-0.06}^{+0.08}$ & $2.43_{-0.29}^{-0.64}$ & $2.16_{-0.18}^{+0.01}$ & $5.10_{-0.52}^{-1.52}$ & 1.01 & 190 & 3.41 & 30.90 \\
\hline & & 6 & $0.51_{-0.12}^{-0.13}$ & $0.74_{-0.05}^{-0.06}$ & $2.01_{-0.038}^{+0.29}$ & $2.23_{-0.31}^{+0.38}$ & $3.27_{-0.45}^{+0.38}$ & 1.31 & 220 & 2.45 & 30.76 \\
\hline & & 7 & $0.28_{-0.16}^{+0.12}$ & $0.83_{-0.15}^{+0.05}$ & $\begin{array}{l}-0.20 \\
1.89_{-043}^{+0.80}\end{array}$ & $\begin{array}{l}-0.31 \\
2.23^{+0.49}\end{array}$ & $\begin{array}{r}-0.45 \\
1.83_{-115}^{+0.63}\end{array}$ & 1.10 & 128 & 1.82 & 30.63 \\
\hline & & 8 & $0.32_{-0.10}^{-0.16}$ & $0.74_{-0.06}^{-0.06}$ & $1.54_{-0.14}^{+0.20}$ & $1.76_{-0.28}^{-0.45}$ & $1.90_{-0.31}^{+0.13}$ & 1.15 & 217 & 1.57 & 30.57 \\
\hline \multirow[t]{13}{*}{$28-100$} & BP Tau & 1 & $0.96_{-0.22}^{+0.24}$ & $0.37_{-0.03}^{+0.04}$ & $1.57_{-0.11}^{+0.11}$ & $0.63_{-0.12}^{+0.14}$ & $0.94_{-0.07}^{+0.05}$ & 1.25 & 226 & 0.63 & 30.17 \\
\hline & & 2 & $1.00^{-0.22}$ & $0.41_{-0.06}^{+0.03}$ & $2.43_{-0.44}^{+0.11}$ & $0.68_{-0.12}^{+0.12}$ & $1.25_{-0.19}^{+0.16}$ & 0.76 & 75 & 0.87 & 30.31 \\
\hline & & 3 & 1.00 & $0.79_{-0.24}^{-0.06}$ & $4.04^{+1.95}$ & $0.78_{-0.24}^{+0.22}$ & $1.93_{-0.31}^{+0.19}$ & 0.98 & 79 & 1.46 & 30.54 \\
\hline & & 4 & $1.26_{-0.13}^{+0.12}$ & $0.75_{-0.06}^{+0.23}$ & $2.65_{-0.17}^{-0.05}$ & $0.89_{-0.16}^{+0.24}$ & $3.29_{-0.21}^{+0.21}$ & 0.93 & 419 & 2.06 & 30.69 \\
\hline & & 5 & $\begin{array}{l}1.30_{-0.13}^{+0.29} \\
1.020\end{array}$ & $0.79_{-0.09}^{+0.06}$ & $\begin{array}{l}-0.17 \\
2.39_{-0.49}^{+0.49}\end{array}$ & $\begin{array}{l}0.05_{-026}^{+0.16} \\
1.28\end{array}$ & $1.93_{-0.33}^{+0.52}$ & 1.23 & 133 & 1.59 & 30.57 \\
\hline & & 6 & $0.85_{-0.27}^{+0.94}$ & $\begin{array}{r}-0.09 \\
0.77_{-0.09}^{+0.08}\end{array}$ & $2.13_{-0.30}^{-0.54}$ & $1.06_{-0.21}^{+0.26}$ & $1.55_{-0.28}^{+0.33}$ & 0.80 & 101 & 1.17 & 30.44 \\
\hline & & 7 & $0.95_{-0.19}^{+2.64}$ & $0.78_{-0.08}^{+0.09}$ & $1.82_{-0.19}^{+0.24}$ & $0.80_{-0.14}^{+0.16}$ & $1.27_{-0.21}^{+0.19}$ & 1.14 & 156 & 0.90 & 30.33 \\
\hline & & 8 & $0.64_{-0.038}^{+0.19}$ & $0.78_{-0.08}^{+0.08}$ & $1.60_{-0.015}^{+0.19}$ & $0.52_{-0.019}^{+0.14}$ & $0.94_{-0.026}^{+0.16}$ & 0.97 & 103 & 0.62 & 30.16 \\
\hline & & 9 & $\begin{array}{r}-0.38 \\
1.27_{-0.20}^{+0.26}\end{array}$ & $0.34_{-0.02}^{+0.08}$ & $1.50_{-0.08}^{+0.15}$ & $0.66_{-0.012}^{+0.19}$ & $0.92_{-0.05}^{+0.26}$ & 0.99 & 345 & 0.63 & 30.17 \\
\hline & & 10 & $1.00^{-0.20}$ & $0.40_{-0.03}^{+0.02}$ & $1.62_{-0.12}^{-0.08}$ & $0.54_{-0.07}^{+0.12}$ & $0.94_{-0.09}^{+0.05}$ & 1.20 & 179 & 0.60 & 30.15 \\
\hline & & 11 & $0.87_{-0.17}^{+0.19}$ & $0.37_{-0.02}^{-0.03}$ & $1.51_{-0.07}^{+0.12}$ & $0.49_{-0.07}^{+0.07}$ & $0.71_{-0.05}^{+0.09}$ & 1.05 & 348 & 0.48 & 30.05 \\
\hline & & 12 & $0.69_{-0.26}^{+0.26}$ & $0.38_{-0.03}^{+0.02}$ & $1.87_{-0.21}^{+0.01}$ & $0.49_{-0.14}^{+0.01}$ & $1.01_{-0.07}^{+0.05}$ & 1.03 & 218 & 0.64 & 30.18 \\
\hline & & 13 & $1.03_{-0.40}^{+0.21}$ & $0.31_{-0.03}^{+0.03}$ & $1.29_{-0.07}^{-0.122}$ & $0.45_{-0.16}^{+0.19}$ & $0.94_{-0.21}^{+0.07}$ & 0.98 & 198 & 0.56 & 30.12 \\
\hline \multirow[t]{5}{*}{ 05-031 } & HD 283810 & 1 & $0.01_{-0.01}^{+0.16}$ & $0.41_{-0.03}^{+0.05}$ & $0.98_{-0.07}^{+0.08}$ & $2.16_{-033}^{+0.45}$ & $1.95_{-0.28}^{+0.28}$ & 1.21 & 165 & 1.66 & 30.22 \\
\hline & & 2 & $0.00_{-0.00}^{+0.01}$ & $0.41_{-0.02}^{+0.03}$ & $0.79_{-0.04}^{+0.07}$ & $\begin{array}{l}-0.33 \\
1.69_{-0.31}^{+0.28}\end{array}$ & $\begin{array}{r}-0.28 \\
1.93_{-026}^{+0.31}\end{array}$ & 1.49 & 420 & 1.48 & 30.17 \\
\hline & & 3 & $0.01^{-0.00}$ & $0.60_{-0.06}^{+0.02}$ & $1.56_{-0.0}^{+0.04}$ & $3.01_{-049}^{+0.41}$ & $\begin{array}{r}-0.26 \\
4.66_{-0.57}^{+0.59}\end{array}$ & 1.07 & 172 & 3.24 & 30.51 \\
\hline & & 4 & 0.01 & $0.69_{-0.04}^{+0.06}$ & $2.27_{-0.72}^{-3.19}$ & $3.53_{-0.78}^{+0.54}$ & $1.72_{-0.68}^{+0.57}$ & 1.18 & 88 & 2.32 & 30.37 \\
\hline & & 5 & 0.01 & $0.19_{-0.08}^{-0.04}$ & $0.75_{-0.13}^{+0.05}$ & $0.96_{-0.52}^{+0.89}$ & $4.00_{-0.47}^{+2.198}$ & 1.32 & 106 & 1.89 & 30.28 \\
\hline \multirow[t]{11}{*}{$16-031$} & $\mathrm{~J} 04195676+27$ & 4 & $0.11_{-0.11}^{+0.14}$ & $\ldots$ & $1.85_{-0.25}^{+0.25}$ & & $3.34_{-0.21}^{+0.21}$ & 1.10 & 110 & 1.48 & 30.54 \\
\hline & & 5 & $0.10^{-0.11}$ & $\cdots$ & $1.65_{-0.11}^{-0.25}$ & $\cdots$ & $1.81_{-0.07}^{+0.01}$ & 1.29 & 91 & 0.78 & 30.26 \\
\hline & & 6 & 0.10 & $\ldots$ & $1.63_{-0.10}^{+0.21}$ & $\ldots$ & $2.35_{-0.02}^{+0.19}$ & 1.50 & 123 & 1.74 & 30.61 \\
\hline & & 7 & 0.10 & $\ldots$ & $3.63_{-0.64}^{+0.10}$ & $\ldots$ & $6.56_{-0.33}^{+0.02}$ & 0.87 & 69 & 3.71 & 30.94 \\
\hline & & 8 & 0.10 & $\ldots$ & $2.38_{-0.37}^{+0.39}$ & $\ldots$ & $9.88_{-0.21}^{+0.83}$ & 1.02 & 68 & 4.80 & 31.05 \\
\hline & & 9 & 0.10 & $\ldots$ & $3.02_{-0.33}^{+0.30}$ & $\ldots$ & $16.20_{-0.02}^{+1.15}$ & 1.12 & 161 & 8.58 & 31.30 \\
\hline & & 10 & 0.10 & $\ldots$ & $2.01_{-0.25}^{+0.43}$ & $\ldots$ & $13.33_{-0.73}^{+0.08}$ & 1.05 & 65 & 6.08 & 31.16 \\
\hline & & 11 & 0.10 & $\ldots$ & $1.92_{-0.25}^{+0.23}$ & $\ldots$ & $11.22_{-0.56}^{+0.56}$ & 1.17 & 78 & 5.05 & 31.07 \\
\hline & & 12 & 0.10 & $\cdots$ & $1.63_{-0.12}^{+0.25}$ & $\cdots$ & $7.06_{-0.09}^{+0.56}$ & 1.51 & 102 & 3.02 & 30.85 \\
\hline & & 13 & 0.10 & $\ldots$ & $1.64_{-0.10}^{-0.12}$ & $\cdots$ & $\begin{array}{r}r_{-0.09} \\
3.81_{-0.16}^{+0.16}\end{array}$ & 1.26 & 100 & 1.63 & 30.58 \\
\hline & & 14 & 0.10 & $\ldots$ & $1.24_{-0.06}^{-0.10}$ & $\ldots$ & $1.58_{-0.09}^{+0.07}$ & 1.47 & 95 & 0.65 & 30.18 \\
\hline \multirow[t]{13}{*}{$22-024$} & HD 285845 & 1 & $0.42_{-0.10}^{+0.10}$ & $0.64_{-0.04}^{+0.03}$ & $2.52_{-0.030}^{+0.54}$ & $3.27_{-0.24}^{+0.31}$ & $3.27_{-0.38}^{+0.33}$ & 0.93 & 295 & 3.00 & 30.66 \\
\hline & & 2 & $0.33_{-0.23}^{+0.10}$ & $0.77_{-0.06}^{-0.04}$ & $3.49_{-128}^{+0.30}$ & $4.09_{-071}^{-0.84}$ & $3.32_{-0.87}^{+1.01}$ & 0.96 & 86 & 3.58 & 30.74 \\
\hline & & 3 & $0.47_{-0.12}^{-0.21}$ & $0.61_{-0.04}^{-0.06}$ & $2.86_{-0.44}^{+0.28}$ & $3.50_{-0.38}^{+0.71}$ & $5.29_{-0.47}^{+0.87}$ & 1.09 & 232 & 4.21 & 30.81 \\
\hline & & 4 & $0.36_{-0.17}^{-0.12}$ & $0.66_{-0.06}^{+0.16}$ & $3.10_{-0.82}^{+1.44}$ & $3.39_{-0.47}^{-0.38}$ & $3.50_{-0.59}^{+0.47}$ & 1.01 & 119 & 3.30 & 30.70 \\
\hline & & 5 & $0.40_{-0.11}^{+0.17}$ & $0.65_{-0.03}^{+0.06}$ & $3.01_{-0.65}^{+0.82}$ & $3.20_{-0.38}^{+0.47}$ & $2.70_{-0.33}^{+0.59}$ & 1.09 & 215 & 2.78 & 30.63 \\
\hline & & 6 & $\begin{array}{l}-0.11 \\
0.39_{-0.09}^{+0.09}\end{array}$ & $0.65_{-0.03}^{+0.03}$ & $\begin{array}{r}-0.65 \\
3.04_{-0.41}^{+0.48}\end{array}$ & $\begin{array}{l}.09_{-0.28}^{+0.28} \\
2.92\end{array}$ & $\begin{array}{r}-0.33 \\
3.62_{-0.31}^{+0.33}\end{array}$ & 0.90 & 339 & 3.19 & 30.69 \\
\hline & & 7 & $0.51_{-0.08}^{-0.09}$ & $0.64_{-0.04}^{-0.03}$ & $1.65_{-0.10}^{-0.41}$ & $2.70_{-0.24}^{+0.41}$ & $3.57_{-0.45}^{+0.31}$ & 0.91 & 368 & 2.67 & 30.61 \\
\hline & & 8 & $0.34^{+0.02}$ & $0.67^{-0.08}$ & $2.48^{-0.14}$ & $3.13^{-0.45}$ & $1.34^{+0.49}$ & 0.71 & 105 & 1.99 & 30.48 \\
\hline & & 9 & $0.32_{-0.12}^{+0.22}$ & $0.68_{-0.03}^{+0.05}$ & $1.82_{-0.027}^{-0.86}$ & $2.73_{-0.24}^{+0.42}$ & $\begin{array}{r}-0.59 \\
1.11_{-0.26}^{+0.24}\end{array}$ & 0.95 & 191 & 1.64 & 30.40 \\
\hline & & 10 & $0.20_{-020}^{+0.12}$ & $0.66_{-0.08}^{+0.03}$ & $1.37_{-1.09}^{+0.39}$ & $2.02_{-0.47}^{-0.24}$ & $1.11_{-0.31}^{+1.36}$ & 0.67 & 65 & 1.32 & 30.30 \\
\hline & & 11 & $0.59^{+0.09}$ & $0.66^{-0.028}$ & $1.92^{-1.0 .29}$ & $2.80^{-0.31}$ & $2.16^{+0.31}$ & 0.94 & 217 & 2.16 & 30.52 \\
\hline & & 12 & $0.38_{-0.10}^{+0.11}$ & $0.71_{-0.04}^{-0.06}$ & $1.84_{-0.05}^{-0.24}$ & $2.61_{-0.25}^{-0.26}$ & $1.36_{-0.40}^{+0.21}$ & 1.04 & 230 & 1.72 & 30.42 \\
\hline & & 13 & $0.35_{-0.06}^{-0.11}$ & $0.66_{-0.02}^{+0.04}$ & $1.56_{-0.13}^{+0.37}$ & $2.38_{-0.09}^{+0.09}$ & $0.89_{-0.09}^{+0.09}$ & 1.10 & 428 & 1.38 & 30.32 \\
\hline
\end{tabular}

${ }^{a}$ Unabsorbed X-ray flux and luminosity in the $0.3-7.3 \mathrm{keV}$ band. $L_{\mathrm{X}}$ is computed for a distance of $140 \mathrm{pc}$, except for HD $283810(d=60 \mathrm{pc})$ and HD $285845(d=90 \mathrm{pc})$. Note that the luminosity for XEST-16-031 is only indicative, since the true distance of this star is not known. 